\title{
Comparative Efficacy and Safety of Chinese Herbal Injections Combined With Cyclophosphamide and 5-Fluorouracil Chemotherapies in Treatment of Breast Cancer: A Bayesian Network Meta-Analysis
}

\author{
Shuyu Liu ${ }^{\dagger}$, Haojia Wang ${ }^{\dagger}$, Miaomiao Wang, Xiaohong Hu, Wenqian Yang, Ruiqi Jin, \\ Yifei Geng, Mengwei Ni, Jiarui Wu * and Xiaomeng Zhang
}

Department of Clinical Chinese Pharmacy, School of Chinese Materia Medica, Beijing University of Chinese Medicine, Beijing, China

\section{OPEN ACCESS}

Edited by:

SubbaRao V. Madhunapantula, JSS Academy of Higher Education and Research, India

Reviewed by: Yun K. Tam

Sinoveda Canada Inc., Canada Subhash Chandra Mandal, Government of West Bengal, India

*Correspondence: Jiarui Wu exogamy@163.com

${ }^{t}$ These authors have contributed equally to this work

Specialty section: This article was submitted to Ethnopharmacology, a section of the journal

Frontiers in Pharmacology

Received: 14 June 2020 Accepted: 18 December 2020 Published: 27 January 2021

Citation:

Liu S, Wang H, Wang M, Hu X, Yang $W$, Jin R, Geng $Y, N i M$, Wu J and Zhang $X$ (2021) Comparative Efficacy and Safety of Chinese Herbal Injections

Combined With Cyclophosphamide and 5-Fluorouracil Chemotherapies in Treatment of Breast Cancer: A

Bayesian Network Meta-Analysis.

Front. Pharmacol. 11:572396

doi: 10.3389/fphar.2020.572396
Background: Given the limitations of chemotherapy for the treatment of breast cancer (BC) and the wide exploration of Chinese herbal injections ( $\mathrm{CH} I \mathrm{~s}$ ), this network meta-analysis (NMA) was conducted to analyze the comparative efficacy and safety of nine $\mathrm{CH}$ ls combined with $\mathrm{CF}$ (Cyclophosphamide and 5-Fluorouracil) chemotherapy regimens in the treatment of BC.

Methods: Several electronic databases were searched to identify randomized controlled trials (RCTs) from inception to January 6, 2020. RCTs were screened by pre-established eligibility criteria, and the quality of which was assessed using the Cochrane risk of bias tool. Outcomes such as the clinical effectiveness rate, performance status, peripheral hemogram, and detection of T-lymphocyte subsets were analyzed using the Winbugs 1.4.3 and Stata 13.0 software. Surface under the cumulative ranking curve (SUCRA) probability values were applied to rank the examined treatments. Cluster analysis was performed to compare the effect of $\mathrm{CHls}$ between two or three different outcomes.

Results: A total of $84 \mathrm{RCT}$ s involving 7855 patients and nine $\mathrm{CH}$ ls were included. The results showed that compared to CF chemotherapy regimens alone, the ones injected along with Aidi, Shenmai, Shenqi Fuzheng, Kangai, Kanglaite, or Shengmai combined with CF can improve the clinical effectiveness rate. Aidi, Shenmai, Shenqi Fuzheng, Compound Kushen, Kangai, and Kanglaite injection combined with CF can improve the performance status. Shenqi Fuzheng injection was considered as a favorable choice for relieving adverse reactions. According to the results of cluster analysis, Aidi injection and Compound Kushen injection plus CF were more favorable for the clinical effectiveness rate and performance status.

Conclusion: In conclusion, Shenqi Fuzheng, Compound Kushen, Aidi, and Kangai injection combined with CF chemotherapy regimen have more significant effects for patients with $\mathrm{BC}$. However, more high-quality clinical RCTs, especialy which correctly use blinding and allocation concealment, are required to support the conclusions.

Keywords: breast cancer, network meta-analysis, bayesian model, randomized controlled trials, Chinese herbal injections 


\section{INTRODUCTION}

Breast cancer (BC) is one of the most common cancers and the main cause of cancer death in women around the world (Siegel et al., 2019). The high morbidity and high mortality of BC pose a huge potential threat to public health in China (Zheng et al., 2013; Fan et al., 2014; Chen et al., 2016). Although significant progress has been made in the fields of surgical treatment, endocrine therapy, chemotherapy, radiotherapy, and targeted therapy for BC in the past few decades, most BC patients still experience symptoms such as cancer metastasis, relapse, and adverse reactions (ADRs) (Wang and Li, 2018). In the chemotherapy of breast cancer, anthracyclines, such as doxorubicin (A), epirubicin (E) and pirarubicin (T), are the most widely used broad-spectrum anti-tumor drugs with a definite clinical effect. Therefore, this study selected CF chemotherapy regimens, especially those with anthracyclines as a combination medicine, as a control group for efficacy evaluation.

As an important part of complementary and alternative medicine, traditional Chinese medicine (TCM) has become one of the main methods of comprehensive anti-cancer treatment due to its advantages in treating complications and preventing drug resistance (Zhou et al., 2017). According to TCM theory, the basic pathogenesis of $\mathrm{BC}$ is meridian block, qi stagnation, blood stasis, etc. (Gao and Wang, 2013). Therefore, nourishing liver and kidney, enhancing the body's resistance and eliminating pathogens were deemed to be the principle of treating BC (Dong and Ren, 2018). Chinese herbal injections (CHIs) has the characteristics of obvious curative effect and high bioavailability (Lai et al., 2012). At present, CHIs combined with chemotherapy has been widely used in the treatment of malignant tumors, but there is still a lack of highquality evidence-based medicine at home and abroad. The preliminary search found that many classic Meta-analysis have been published before to evaluate the safety and effectiveness of a single kind of $\mathrm{CHI}$ as an adjuvant treatment of breast cancer. However, they cannot horizontally compare and rank the curative effects of various CHIs. And some meta-analysis does not reach required information size to provide convincing results. Bayesian network meta-analysis (NMA) can have the advantages of combining direct and indirect evidence to compare multiple interventions. This method can increase the credibility of the evidence and select the optimal CHIs for the BC treatment. Therefore, this study used a NMA method to comprehensively evaluate the efficacy and safety of CHIs combined with $\mathrm{CF}$ (Cyclophosphamide and 5-Fluorouracil) chemotherapy regimen against $\mathrm{BC}$.

\section{METHODS}

This study is reported in strict accordance with the standard format of the Preferred Reporting Items for Systematic Reviews and Meta-Analysis Specification: PRISMA Extension Statement specification (Hutton et al., 2015; Ge et al., 2017).

\section{Search Strategy}

A computerized search of PubMed, the Cochrane library, embase, the China National Knowledge Infrastructure database (CNKI),
Wanfang database, the China Science and Technology Journal database (VIP), and SinoMed databases for literatures on RCTs of CHIs in the treatment of BC was performed up to January 6, 2020. The searching strategy was developed with reference to the Cochrane Handbook for Systematic Reviewers (version 5.1.0). The search terms were divided into three parts: CHIs, BC, and RCTs. The varieties of CHIs searched included Shenqi Fuzheng injection (SQFZI), Compound Kushen injection (CKI), Shenmai injection (SMI), Kangai injection (KAI), Aidi injection (ADI), Kanglaite injection (KLTI), Huangqi injection (HQI), Huachansu injection (HCSI), and Shengmai injection (SI). The detailed search strategy taking PubMed as an example is described in Presentation File.

\section{Inclusion Criteria Types of studies}

Randomized controlled trials (RCTs) regarding CHIs combined with $\mathrm{CF}$ in the treatment of $\mathrm{BC}$ were eligible, which is referred to as "random", with or without blinding.

\section{Types of Participants}

All patients were diagnosed with BC pathologically and histologically, no limitation on gender and nationality.

\section{Types of Interventions}

Patients in control group only received CF chemotherapy regimens, including $\mathrm{CAF}, \mathrm{CEF}, \mathrm{CTF}, \mathrm{CMF}, \mathrm{FAC}, \mathrm{FEC}$, etc., (C: cyclophosphamide; F: 5-fluorouracil; A: doxorubicin; T: pirarubicin; E: epirubicin; $\mathrm{M}$ : methotrexate). Patients in treatment group received $\mathrm{CHI}$ with $\mathrm{CF}$ therapy.

\section{Types of Outcomes}

Primary outcomes include clinical effectiveness rate, performance status, T-lymphocyte subsets (including CD3 +, CD4 +, CD8 +, $\mathrm{CD} 4+/ \mathrm{CD} 8+$ ), peripheral hemogram including white blood cells (WBC) and platelets (PLT), and ADRs. Secondary outcomes include tumor markers, the Karnofsky Performance Score (KPS), ECG changes, comparison of LVEF, liver and kidney function, and myocardial enzyme spectrum. According to the WHO Objective Response Criteria in Solid Tumors, The clinical effectiveness rate $=$ [number of complete response $(\mathrm{CR})$ patients + partial response $(\mathrm{PR})] /$ total number of patients $\times 100 \%$. In accordance with KPS functional status scoring criteria, there are three levels: improvement (KPS score increased by more than 10 points), stability (KPS score changed by less than 10 points) and decrease (KPS score decreased by more than 10 points). An increase of more than 10 points in KPS score is considered as a significant improvement in performance status. RCTs that have at least any one of the primary outcome indexes were included in this study.

\section{Exclusion Criteria}

The exclusion criteria were as follows: 1) There are other TCM treatment methods except CHIs in the chemotherapy regimen; 2) For the repeatedly published articles, only remained the latest or more comprehensive ones; 3 ) Researches with incomplete data or obvious errors; 4) The article could not be obtained. 


\section{Data Extraction and Quality Assessment} NoteExpress software was used to eliminate duplicate RCTs. Two researchers independently read the title and abstract of RCTs, and obviously irrelevant literatures were screened out. Then, the remaining articles were read in full according to the inclusion and exclusion criteria for rescreening. In case of any disagreement, it may be handed over to a third party for judgment. The following information were recorded from the included RCTs: the first author, publication year, patient characteristics (sample size, age, disease duration, TNM stage), specific chemotherapy regimen, usage and dosage of CHIs, course of treatment, outcomes, type of studies and the domains of risk of bias.

Two researchers evaluated the risk of bias of included RCTs using the Cochrane Handbook for Systematic Reviewers (version 5.1.0), RCT risk of bias assessment tool. Evaluation indicators include: 1) sequence generation (selection bias); 2) allocation concealment (selection bias); 3) blinding of patients and personnel (performance bias); 4) blinding of outcome assessors (detection bias); 5) incomplete outcome data (attrition bias); 6) elective reporting (reporting bias); 7) other bias. Each indicator contains three levels: low risk, unclear and high risk. Disagreement during the evaluation process may be referred to a third party for determination.

\section{Data Analysis}

WinBUGS 1.4.3 software was used for statistical analysis of data. Binary outcomes were calculated as odds ratio (OR); continuous outcomes were calculated as weighted mean difference (MD), along with $95 \%$ confidence intervals (95\% CIs) for effect sizes. OR excluding one or MD values excluding 0 were considered as statistically significant (Liu, 2012). Stata 13.0 software was applied to draw cumulative probability ranking plots, reticular relationship plots, and funnel plots for each intervention. The cumulative probability ranking plot can simultaneously obtain the surface under the cumulative ranking area curve (SUCRA). SUCRA with $100 \%$ indicated that the intervention was absolutely effective, and $0 \%$ indicated that the intervention was absolutely ineffective (Chen and Chen, 2013; Jia et al., 2015). Cluster analysis resulted in relatively better interventions in both cluster measures (Bian et al., 2011; Wu et al., 2017). In the network graph, the dot area represented the number of patients with relevant interventions, and the thickness of the line between each point represented the number of included studies (Leucht et al., 2013; Li et al., 2014). In the funnel plot, evenly distributed points indicated that the publication bias of the included RCTs was small (Gao et al., 2018). In addition, if there was a closed loop of intervention in the study, a consistency test was required. The results were expressed as $p$ value, IF (inconsistency factor) and 95\% CIs. If $p$ value was greater than 0.05 and IF value was close to 0 , the corresponding direct comparison evidence was consistent with indirect comparison evidence (Jinatongthai et al., 2017).

\section{RESULTS}

\section{Search Results}

As Figure 1 shown, a total of 1177 RCTs were retrieved according to the established searching strategies. After the exclusion of duplications and irrelevant studies, 170 RCTs were remained. After screening on full-text level, 86 RCTs were excluded for the following reasons: 1$)$ not an RCT $(n=5) ; 2)$ Intervention was not met the inclusion criteria $(n=4) ; 3)$ disease was not $\mathrm{BC}(n=11)$; 4) Outcomes were not in accordance with the inclusion criteria $(n=59)$; 5) duplicated data $(n=7)$. A total of 84 papers were eventually included, all of which were conducted in China from 1999 to 2019.

\section{Inclusion Studies and Characteristics}

A total of 84 RCTs were finally included, including nine CHIs and 7855 female patients (4025 patients in the experimental group and 3830 patients in the control group), whose age ranged from 26 to 66 years old. The nine CHIs involved were ADI (9 RCTs), SMI (12 RCTs), SQFZI (33 RCTs), CKI (8 RCTs), HCSI (2 RCTs), HQI (2 RCTs), KAI (2 RCTs), KLTI (5 RCTs), and SI (1 RCTs). All of the nine CHIs were given via intravenous drip once a day, most of which were treated with a course of 21 days. Detailed information of included RCTs are shown in Table 1. The relationship of outcome indicators is shown in Figure 2.

\section{METHODOLOGICAL QUALITY}

There were 22 studies of 84 included studies were grouped according to the method of random number tables, five studies described a method of randomization including draw lots or the envelope, two studies used a method of randomization including coin toss, and one study was divided into groups by stratified random method. The above 30 studies were rated as "low risk" regarding sequence generation. Three studies were evaluated as "high risk", because they grouped according to the time of admission. The risk of remaining studies was deemed "unclear", because they just mentioned "randomization". One study adopted double-blind randomized clinical trial and evaluated for "low risk" regarding performance bias. The attrition bias of all studies were evaluated as "low risk" due to the lack of incomplete data. The risk of bias entries for the rest studies were rated as "unclear" due to insufficient information. Results are shown in Figure 3.

\section{Network Meta-Analysis Clinical Effectiveness Rate}

As the main outcome index, the clinical effectiveness rate directly reflected the curative effect of patients. A total of 47 studies referred to the clinical effectiveness rate, involving nine $\mathrm{CHI}$ and 10 interventions. There were seven studies on ADI combined with CF, three studies on SMI combined with CF, 18 studies on SQFZI combined with CF, three studies on CKI combined with CF, two studies on HCSI combined with CF, two studies on HQI combined with $\mathrm{CF}$, eight studies on AKI combined with $\mathrm{CF}$, three studies on KLTI combined with CF, and one study on SI combined with CF. ORs showed that comparing to using $\mathrm{CF}$ alone, combined with ADI (OR $=0.43,95 \%$ CIs: $0.30-0.75)$, SMI (OR $=0.47,95 \%$ CIs: $0.27-0.93)$, SQFZI $(\mathrm{OR}=0.45$, 95\%CIs: $0.33-0.55)$, KAI (OR $=0.43$, 95\%CIs: $0.31-0.65)$, KLTI (OR = $0.27,95 \%$ CIs: $0.15-0.57)$, SI (OR $=0.18,95 \%$ CIs: $0.03-0.48)$ on 


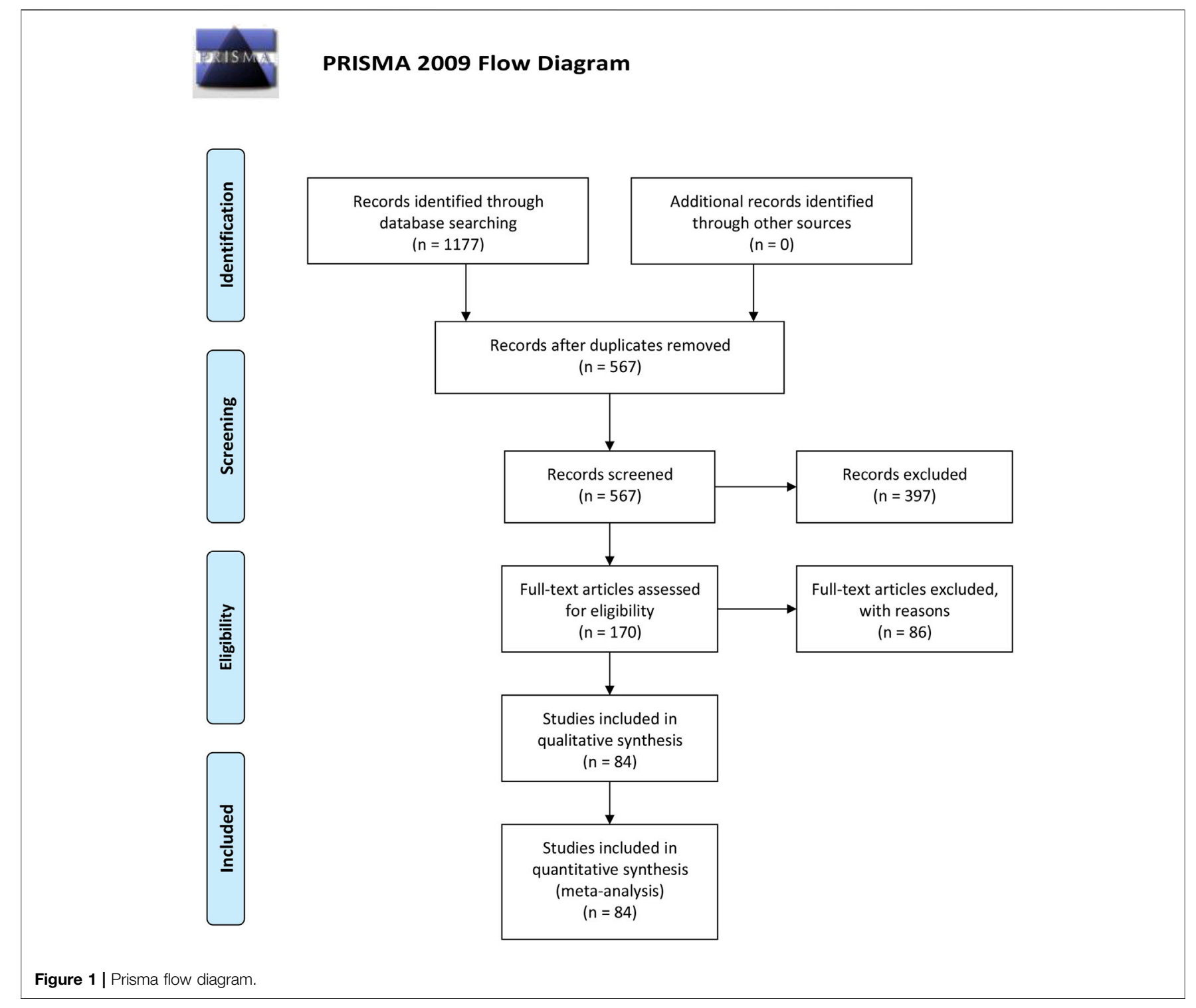

the basis of chemotherapy can improve the clinical effectiveness rate and make the difference between groups statistically significant. Specific values are shown in Table 2.

After the ranking of each intervention efficacy, the combination of CF and SI (88.9\%) had the highest probability of being the best treatment for $\mathrm{BC}$ in terms of improving the clinical effectiveness rate, followed by the combination of CF and KLTI (83.6\%) and the combination of CF and ADI (59.2\%). The ranking results of interventions are shown in Figure 4, and the SUCRA values are shown in Table 3.

\section{Performance Status}

A total of 30 studies reported performance status, involving seven $\mathrm{CHI}$ and eight intervenions. Three of the studies regarding ADI combined with $\mathrm{CF}$, five regarding SMI combined with $\mathrm{CF}, 11$ regarding $\mathrm{CKI}$ combined with $\mathrm{CF}$, two regarding $\mathrm{CKI}$ combined with $\mathrm{CF}$, one regarding $\mathrm{HQI}$ combined with $\mathrm{CF}$, six regarding
KAI combined with CF, and two of the studies regarding KLTI combined with $\mathrm{CF}$. ORs showed that comparing to using $\mathrm{CF}$ alone, combined with ADI (OR = 0.21, 95\%CIs: 0.09-0.47), SMI $(\mathrm{OR}=0.32$, 95\%CIs: $0.20-0.55)$, SQFZI $(\mathrm{OR}=0.29$, 95\%CIs: $0.20-0.39)$, CKI (OR $=0.45,95 \%$ CIs: $0.20-0.95)$, KAI (OR $=0.31$, 95\%CIs: $0.16-0.52)$ as well as KLTI (OR $=0.27$, 95\%CIs: $0.10-0.69$ ) on the basis of chemotherapy can improve the performance status and make the difference between groups statistically significant. Specific values are shown in Table 2.

After the ranking of each intervention efficacy, the combination of $\mathrm{CF}$ and $\mathrm{ADI}(81.3 \%)$ had the highest probability of being the best treatment for $\mathrm{BC}$ in terms of improving the performance status, followed by the combination of CF and KLTI (63.1\%) and the combination of CF and SQFZI (62.9\%). The ranking results of interventions are shown in Figure 4, and the SUCRA values are shown in Table 3. 
TABLE 1 | Characteristics of the studies included in this meta-analysis.

\begin{tabular}{|c|c|c|c|c|c|c|c|c|c|c|}
\hline \multirow[t]{2}{*}{ Study ID } & \multirow[t]{2}{*}{ Cases (E/C) } & \multirow[t]{2}{*}{ Average age } & \multicolumn{4}{|c|}{ TNM clinical stage (E/C) } & \multirow[t]{2}{*}{ Treatment group intervention } & \multirow[t]{2}{*}{ Control group intervention } & \multirow[t]{2}{*}{ Course } & \multirow[t]{2}{*}{ Outcomes } \\
\hline & & & 1 & II & III & IV & & & & \\
\hline Song et al. (2006) & $48 / 48$ & $\begin{array}{l}\text { E: } 51 \\
\text { C: } 50\end{array}$ & \multicolumn{4}{|c|}{ NR } & $\mathrm{ADI} 50 \mathrm{ml}+\mathrm{CAF} / \mathrm{TA}$ & $\mathrm{CAF} / \mathrm{TA}$ & $21 d \times 2$ & (1) (5) \\
\hline Yang (2005) & $30 / 30$ & $\begin{array}{l}\text { E: } 48.4 \\
\text { C: } 47.6\end{array}$ & / & / & $17 / 18$ & $12 / 13$ & $\mathrm{ADI} 100 \mathrm{ml}+\mathrm{CAF}$ & CAF & $21 d \times 2$ & (1) (2) (3) \\
\hline Chen WM (2016) & $39 / 40$ & $\begin{array}{l}\text { E: } 46.73 \pm 14.29 \\
\text { C: } 45.98 \pm 15.78\end{array}$ & / & / & $29 / 28$ & $10 / 12$ & ADI $100 \mathrm{ml}+\mathrm{CEF}$ & CEF & $21 d \times 6$ & (2) (3) (5) (6) \\
\hline Dang and Wang (2010) & $28 / 20$ & $\begin{array}{l}E: 36.2 \pm 3.6 \\
\text { C: } 37.5 \pm 4.2\end{array}$ & $5 / 4$ & $19 / 14$ & $4 / 2$ & / & ADI $100 \mathrm{ml}+\mathrm{CTF}$ & CTF & $21 d \times 3$ & (1) (4) (5) (7) (10) \\
\hline Liu et al. (2005) & $50 / 50$ & 45 & \multicolumn{4}{|c|}{$\|-I I I$} & ADI $100 \mathrm{ml}+\mathrm{CEF}$ & CEF & $21 d \times 2$ & (1) (3) (5) (6) \\
\hline Li and Gong (2006) & $32 / 20$ & $\begin{array}{l}E: 46.2 \pm 2.6 \\
\text { C: } 44.5 \pm 3.2\end{array}$ & $5 / 3$ & $22 / 14$ & $5 / 3$ & / & ADl $100 \mathrm{ml}+\mathrm{CEF}$ & CEF & $21 d \times 3$ & (1) (5) (7) (10) \\
\hline Ye et al. (2002) & $18 / 17$ & $\begin{array}{l}\text { E: } 47.2 \\
\text { C: } 47\end{array}$ & \multicolumn{4}{|c|}{ NR } & $\mathrm{ADI} 100 \mathrm{ml}+\mathrm{CAF}$ & CAF & $15 d$ & (1) (2) (5) \\
\hline Ren and Zhang (2005) & $60 / 40$ & 39.5 & \multicolumn{4}{|c|}{ II-III } & $\mathrm{ADI} 80 \mathrm{ml}+\mathrm{CMF}$ & $\mathrm{CMF}$ & $21 d \times 2$ & (1) (5) \\
\hline Han et al. (2014) & $64 / 64$ & $46.7 \pm 20.3$ & \multicolumn{4}{|c|}{$N R$} & ADI $100 \mathrm{ml}+\mathrm{CEF}$ & CEF & $21 d \times 6$ & (3) (5) \\
\hline Xiong et al. (2006) & $40 / 43$ & $\begin{array}{l}\text { E: } 31-69 \\
\text { C: } 33-71\end{array}$ & \multicolumn{4}{|c|}{$N R$} & $\mathrm{SMl} 30 \mathrm{ml}+\mathrm{CAF}$ & CAF & $10 d$ & (8) \\
\hline Wang et al. (2012) & $31 / 30$ & $\begin{array}{l}\text { E: } 57.5+8.3 \\
\text { C: } 60.3+6.9\end{array}$ & \multicolumn{4}{|c|}{ NR } & SMl $100 \mathrm{ml}+\mathrm{CAF}$ & CAF & $21 d$ & (8) \\
\hline Yao et al. (2016) & $49 / 49$ & $30-65$ & \multicolumn{4}{|c|}{ NR } & SMI $60 \mathrm{ml}+\mathrm{CEF}$ & CEF & $21 d \times 6$ & (9) (10) \\
\hline Chen et al. (2018) & $45 / 45$ & $\begin{array}{l}\text { E: } 46.08 \pm 4.90 \\
\text { C: } 45.38 \pm 4.97\end{array}$ & $12 / 10$ & $21 / 23$ & $12 / 12$ & / & $\mathrm{SMl} 50 \mathrm{ml}+\mathrm{CTF}$ & CTF & $21 d \times 6$ & (1) (10) \\
\hline Liu et al. (2014) & $22 / 20$ & $\begin{array}{l}E: 50.18 \pm 7.06 \\
C: 48.62 \pm 6.81\end{array}$ & \multicolumn{4}{|c|}{ NR } & SMl $100 \mathrm{ml}+$ FAC & FAC & $21 d \times 4$ & (8) (9) \\
\hline Zhang (2013) & $40 / 40$ & $\begin{array}{l}\text { E: } 26-62 \\
\text { C: } 31-65\end{array}$ & / & $4 / 5$ & $22 / 22$ & $14 / 13$ & SMl $60 \mathrm{ml}+\mathrm{CTF}$ & CTF & $21 d \times 2$ & (1) (2) (3) (5) (7) \\
\hline Huang et al. (2009) & $30 / 30$ & $\begin{array}{l}E: 41.2 \\
\text { C: } 42.3\end{array}$ & \multicolumn{4}{|c|}{ NR } & SMl $60 \mathrm{ml}+\mathrm{CTF}$ & CTF & $21 d \times 2$ & (1) (2) (3) (5) \\
\hline Lao et al. (2011) & $30 / 30$ & E: 52.4 C: 50.8 & / & / & $13 / 14$ & $17 / 16$ & $\mathrm{SMl} 40 \mathrm{ml}+\mathrm{CAF}$ & CAF & $14 d \times 2$ & (2) (3) (5) \\
\hline Chen et al. (2010) & $30 / 30$ & E: 46 C: 42 & \multicolumn{4}{|c|}{ NR } & SMl $100 \mathrm{ml}+\mathrm{CAF}$ & CAF & $21 d \times 3$ & (2) (3) (5) \\
\hline Liu et al. (2009) & $30 / 30$ & E: 47 C: 46 & I & / & $11 / 13$ & $19 / 17$ & SMl $60 \mathrm{ml}+\mathrm{CAF}$ & CAF & $28 d \times 3$ & (2) (3) (5) \\
\hline Bu and Zhang (2018) & $42 / 41$ & 48.3 & \multicolumn{4}{|c|}{ NR } & SMl $50 \mathrm{ml}+\mathrm{CTF}$ & CTF & $21 d \times 6$ & (9) (10) \\
\hline Yang (2008) & $461 / 436$ & NR & \multicolumn{4}{|c|}{$\mathrm{NR}$} & SMl $50 \mathrm{ml}+\mathrm{CAF}$ & CAF & $21 d \times 6$ & (5) (8) (10) \\
\hline Wang F (2015) & $47 / 45$ & E: $42.85 \pm 3.11$ C: $41.96 \pm 3.88$ & / & 57 & 35 & / & SQFZI $250 \mathrm{ml}+\mathrm{CAF}$ & CAF & $21 d \times 2$ & (1) (7) \\
\hline Liu (2017) & $52 / 52$ & $\begin{array}{l}\text { E: } 41.58 \pm 3.23 \\
\text { C: } 40.41 \pm 3.72\end{array}$ & \multicolumn{4}{|c|}{ NR } & SQFZI $250 \mathrm{ml}+\mathrm{CAF}$ & CAF & $21 d \times 3$ & (1) (5) 7 \\
\hline Qi et al. (2013) & $26 / 20$ & 52 & \multicolumn{4}{|c|}{ NR } & SQFZI $250 \mathrm{ml}+\mathrm{CAF}$ & CAF & $21 d \times 2$ & (1) (4) \\
\hline Zou et al. (2006) & $32 / 32$ & 52.5 & \multicolumn{4}{|c|}{ NR } & SQFZI + CTF & CTF & $14 d$ & (3) \\
\hline Lu et al. (2010) & $58 / 52$ & 48.5 & \multicolumn{4}{|c|}{ NR } & SQFZI $250 \mathrm{ml}+$ CAF & CAF & $21 d \times 3$ & (2) (4) \\
\hline Diao et al. (2018) & $47 / 47$ & $\begin{array}{l}\text { E: } 51.68 \pm 9.36 \\
\text { C: } 52.94 \pm 10.14\end{array}$ & $14 / 15$ & $23 / 21$ & $10 / 11$ & / & SQFZI $250 \mathrm{ml}+\mathrm{FEC}$ & FEC & $21 d \times 6$ & (1) (3) \\
\hline Song (2004) & $21 / 25$ & $\begin{array}{l}\text { E: } 52 \\
\text { C: } 58\end{array}$ & l & $18 / 22$ & $3 / 3$ & ' & SQFZI $250 \mathrm{ml}+\mathrm{CMF}$ & $\mathrm{CMF}$ & $14 \mathrm{~d} \times 2$ & (2) (5) (6) \\
\hline Chen and Lin (2007) & $34 / 34$ & 51 & \multicolumn{4}{|c|}{$N R$} & SQFZI $250 \mathrm{ml}+\mathrm{CEF}$ & CEF & $21 d \times 6$ & (4) (5) \\
\hline Yang (2016) & $40 / 40$ & $\begin{array}{l}E: 42.12 \pm 1.33 \\
C: 42.89 \pm 1.21\end{array}$ & $16 / 16$ & $16 / 15$ & $8 / 9$ & ' & SQFZI $250 \mathrm{ml}+\mathrm{CEF}$ & CEF & $28 d \times 2$ & (1)(3) (5) \\
\hline Liu and Song (2017) & $80 / 80$ & $\begin{array}{l}E: 45.36 \pm 3.37 \\
C: 46.21 \pm 9.83\end{array}$ & \multicolumn{4}{|c|}{ NR } & SQFZI $250 \mathrm{ml}+\mathrm{CAF}$ & CAF & $21 d$ & (1) (7) \\
\hline
\end{tabular}


TABLE 1 | (Continued) Characteristics of the studies included in this meta-analysis,

Cases (E/C)

Average age

TNM clinical stage (E/C)

Treatment group intervention

Control group intervention Course Outcomes

Zhang et al. (2018)

$64 / 64$

E: $49.49 \pm 18.21$

Guo and Hu (2018)

$46 / 46$

C: $49.60 \pm 18.36$

$$
\begin{aligned}
& \text { E: } 33-66 \\
& \text { C: } 32-64
\end{aligned}
$$

Li et al. (2015)

$80 / 80$

Liang et al. (2014)

Jia et al. (2016)

Xie (2014)

E: $48.53 \pm 5.17$

C: $48.42 \pm 5.13$

$45.8 \pm 2.3$

E: $45.35 \pm 10.02$

C: $43.78 \pm 9.18$

E: $54.4 \pm 3.8$

C: $52.8 \pm 4.3$

Xiang (2019)

E: $45.26 \pm 10.68$

C: $45.32 \pm 10.52$

Huang et al. (2008)

E: 47

Li S (2018)

Xiao (2005)

Sun and Zheng (2005)

E: $41.2 \pm 1.5$

C: $41.5 \pm 1.5$

56.7
E: 58

C: 60

Dai et al. (2007)

$43 / 39$

E: $45.5 \pm 26.8$

C: $46.1 \pm 27.5$

E: $42.65 \pm 8.27$

C: $42.63 \pm 8.24$

E: $41.75 \pm 2.77$

$\mathrm{He}$ (2015)

$38 / 38$

C: $40.85 \pm 5.12$

Mi (2011)

$50 / 50$

Ma et al. (2015)

Wang W (2015)

Wang (2013)

Fu (2014)

Yuan et al. (2008)

Wang et al. (2006)

Yang et al. (2007)

Zhu et al. (2008)

Wang and Liu (2007)

Zhang and Zhang (2019)

E: 42

C: 45

$36 / 36$

$65 / 65$

NR

E: $46.3 \pm 4.6$

C: $47.4 \pm 5.2$

E: $45.5 \pm 9.8$

C: $45.2 \pm 9.8$ 32-52

$45 / 45$

$38 / 35$

$40 / 32$

NR

E: $45.2 \pm 9.8$

C: $46.7 \pm 10.5$

$58 / 52$

E: 48

C: 49

E: 52.5

C: 51
42.5

$30 / 30$

$45 / 45$

E: $41.21 \pm 1.02$

C: $41.25 \pm 1.45$

E: $42.7 \pm 10.5$

C: $43.5 \pm 11.2$

Sun (2009)

$61 / 62$

49.6

$29 / 30$
$62 / 60$

39-65

$\begin{array}{llll}\text { I II III IV } & \end{array}$

SQFZl $250 \mathrm{ml}+\mathrm{CAF}$

SQFZI $250 \mathrm{ml}+\mathrm{CAF}$

SQFZI $250 \mathrm{ml}+\mathrm{CAF}$

SQFZI $250 \mathrm{ml}+$ CTF

SQFZl $250 \mathrm{ml}+\mathrm{CAF}$

SQFZI $250 \mathrm{ml}+$ CAF

SQFZl $250 \mathrm{ml}+\mathrm{CAF}$

SQFZl $250 \mathrm{ml}+$ CTF

SQFZI $250 \mathrm{ml}+\mathrm{CAF}$

SQFZI $250 \mathrm{ml}+$ FEC

SQFZl $250 \mathrm{ml}+$ CTF

SQFZl $250 \mathrm{ml}+$ CEF

SQFZl $250 \mathrm{ml}+\mathrm{CAF}$

SQFZl $250 \mathrm{ml}+\mathrm{CF}$

$\begin{array}{lllll}4 / 3 & 19 / 21 & 4 / 3 & 3 / 3 & \text { SQFZl } 250 \mathrm{ml}+\mathrm{AC} / \mathrm{CAF} / \mathrm{EC} / \mathrm{CEF} / \mathrm{TA} / \mathrm{TE}\end{array}$

AC/CAF/EC/CEF/TATTE

SQFZI $250 \mathrm{ml}+\mathrm{CAF}$

SQFZl $250 \mathrm{ml}+\mathrm{CAF}$

SQFZI $250 \mathrm{ml}+\mathrm{CAF}$

SQFZI $250 \mathrm{ml}+$ FAC

SQFZI $250 \mathrm{ml}+$ CAF

SQFZI $250 \mathrm{ml}+$ CEF

SQFZI $250 \mathrm{ml}+\mathrm{CAF}$

SQFZl $251 \mathrm{ml}+$ CEF

$\mathrm{CKI} 20 \mathrm{ml}+\mathrm{CTF}$

$\mathrm{CKI} 20 \mathrm{ml}+\mathrm{CAF}$

$\mathrm{CKI} 20 \mathrm{ml}+\mathrm{CAF}$

$\mathrm{CKI} 30 \mathrm{ml}+\mathrm{CAF}$

$\mathrm{CKI} 30 \mathrm{ml}+\mathrm{CAF}$

CAF

CAF

$21 d \times 2$ (1) (2) (4)

$21 d \times 6$

CAF

CTF

CAF

CAF

CAF

CTF

CAF

FEC

CTF

CEF

CAF

CF

$21 d \times 6$

$21 d \times 2$ (1) (2) (5)

$21 d \times 2$
$21 d \times 2$

$21 d \times 3 \quad$ (1) (5)

$1 d \times 2$ (1)(4) (5)

$21 d \times 2$ (1)(2) (3) (5)

$21 d \times 3 \quad$ (1) (4) (7)

$\mathrm{NR}$ (2) (3) (4)

$21 d \times 2$ (2) (4)

$28 d \times 2 \quad$ (1) (3) (5)

$1 d \times 2$ (1) (3) (5)

21d (1)

$21 d \times 4$

$\mathrm{CAF}$

CAF

$21 d \times 3 \quad$ (1) (2) (3) (5)

$21 d \times 3 \quad$ (2) (4)

CAF $21 d \times 3 \quad$ (1) (5)(7)

FAC

CA

CEF

$21 d \times 3$

NR (3)

NR (2)

$11 / 9 \quad 11 / 16 \quad 5 / 4$

NR
NR

CAF

$21 d \times 3$

CEF

CTF
CAF

$21 d \times 4$

$3 / 1 \quad 48 / 53 \quad 10 / 8 \quad /$

NR
NR

$\mathrm{CAF}$

CAF
CAF 
TABLE 1 | (Continued) Characteristics of the studies included in this meta-analysis.

\begin{tabular}{|c|c|c|c|c|c|c|c|c|c|c|}
\hline \multirow[t]{2}{*}{ Study ID } & \multirow[t]{2}{*}{ Cases (E/C) } & \multirow[t]{2}{*}{ Average age } & \multicolumn{4}{|c|}{ TNM clinical stage (E/C) } & \multirow[t]{2}{*}{ Treatment group intervention } & \multirow[t]{2}{*}{ Control group intervention } & \multirow[t]{2}{*}{ Course } & \multirow[t]{2}{*}{ Outcomes } \\
\hline & & & 1 & II & III & IV & & & & \\
\hline Li (2012) & $30 / 30$ & $49.3 \pm 0.9$ & / & 8 & 31 & 21 & CKI $15 \mathrm{ml}+\mathrm{CTF}$ & CTF & $21 d \times 2$ & (1)(5) (7) \\
\hline Wei (2010) & $12 / 12$ & 52 & \multicolumn{4}{|c|}{ NR } & CKI $20 \mathrm{ml}+\mathrm{CTF}$ & CTF & $21 d \times 2$ & (3) (7) (8) \\
\hline Gu et al. (2015) & $45 / 44$ & $\begin{array}{l}\text { E: } 47.2 \pm 2.4 \\
\text { C: } 47.8 \pm 3.1\end{array}$ & $12 / 13$ & $19 / 18$ & $14 / 13$ & / & $\mathrm{CKI} 15 \mathrm{ml}+\mathrm{FEC}$ & FEC & $21 d$ & (1) \\
\hline Pan (2011) & $80 / 80$ & 56 & \multicolumn{4}{|c|}{ IIII-IV } & $\mathrm{HCSI} 20 \mathrm{ml}+\mathrm{CAF}$ & CAF & $14 d \times 2$ & (1) (5) \\
\hline Song (2002) & $26 / 21$ & 50 & / & $12 / 16$ & 9/8 & $1 / 1$ & HCSI $20 \mathrm{ml}+\mathrm{CAF}$ & CAF & $28 d$ & (1) (5) \\
\hline Lu (2010) & $30 / 30$ & $\begin{array}{c}E: 46.2 \pm 9.3 \\
\text { C: } 44.31 \pm 1.1\end{array}$ & \multicolumn{4}{|c|}{ NR } & $\mathrm{HQl} 40 \mathrm{ml}+\mathrm{CAF}$ & CAF & $14 d \times 2$ & (1) (5) \\
\hline Huang et al. (2013) & $30 / 30$ & $\begin{array}{l}\text { E: } 47.8 \pm 3.2 \\
\text { C: } 51.2 \pm 3.5\end{array}$ & \multicolumn{4}{|c|}{ NR } & $\mathrm{HQl} 40 \mathrm{ml}+\mathrm{CEF}$ & CEF & $21 d \times 2$ & (1) (2) (3) (5) \\
\hline Shi et al. (2017) & $30 / 30$ & $\begin{array}{l}E: 41.83 \pm 4.31 \\
\text { C: } 42.04 \pm 4.25\end{array}$ & $1 / 2$ & $23 / 21$ & $6 / 7$ & / & $\mathrm{KAl} 30 \mathrm{ml}+\mathrm{CAF}$ & CAF & $21 d \times 3$ & (3) (4) (5) \\
\hline Su et al. (2016) & $53 / 53$ & $35-70$ & \multicolumn{4}{|c|}{ NR } & KAl $60 \mathrm{ml}+\mathrm{CTF}$ & CTF & $28 d \times 3$ & (1) (5) \\
\hline Tan et al. (2018) & $30 / 30$ & $\begin{array}{l}\text { E: } 54.1 \pm 6.2 \\
\text { C: } 51.2 \pm 4.6\end{array}$ & / & $19 / 19$ & $11 / 11$ & / & KAl $60 \mathrm{ml}+\mathrm{CTF}$ & CTF & $21 d \times 3$ & (1) (2) (5) \\
\hline Chen FW (2016) & $25 / 25$ & $\begin{array}{l}E: 47.52 \pm 10.31 \\
C: 48.06 \pm 10.28\end{array}$ & \multicolumn{4}{|c|}{ NR } & KAl $60 \mathrm{ml}+\mathrm{TXT}+\mathrm{CEF}$ Sequential & TXT+CEF Sequential & $21 d$ & (1) (2) (5) \\
\hline Li et al. (2006) & $42 / 40$ & $\begin{array}{l}\text { E: } 58 \\
\text { C: } 60\end{array}$ & \multicolumn{4}{|c|}{ NR } & KAl $40 \mathrm{ml}+\mathrm{CTF}$ & CTF & $21 d \times 2$ & (1) (2) \\
\hline Wu and Wu (2011) & $30 / 30$ & $\begin{array}{l}\text { E: } 51.5 \\
\text { C: } 50.5\end{array}$ & \multicolumn{4}{|c|}{ NR } & $\mathrm{KAl} 40 \mathrm{ml}+\mathrm{CTF}$ & CTF & $21 d \times 2$ & (1) (2) (5) \\
\hline Qiu (2016) & $60 / 60$ & $\begin{array}{l}E: 45.8 \pm 10.3 \\
\text { C: } 46.7 \pm 10.8\end{array}$ & / & $48 / 46$ & $12 / 14$ & / & $\mathrm{KAl} 40 \mathrm{ml}+\mathrm{CEF}$ & CEF & $21 d \times 4$ & (1) (3) (5) \\
\hline Pan et al. (2008) & $15 / 15$ & $\begin{array}{l}\text { E: } 56.62 \\
\text { C: } 59.74\end{array}$ & \multicolumn{4}{|c|}{ NR } & $\mathrm{KAl} 60 \mathrm{ml}+\mathrm{CEF}$ & CEF & $21 d$ & (2) (3) (5) \\
\hline Wu (2010) & $49 / 47$ & $\begin{array}{l}\text { E: } 52.2 \pm 11.2 \\
\text { C: } 50.7 \pm 10.5\end{array}$ & / & $37 / 37$ & $12 / 10$ & / & $\mathrm{KAl} 40 \mathrm{ml}+\mathrm{CAF}$ & CAF & $21 d \times 4$ & (2) (4) \\
\hline Cao et al., 2009 & $156 / 80$ & 45 & \multirow{2}{*}{\multicolumn{4}{|c|}{$\begin{array}{l}\| I-I I I \\
N R\end{array}$}} & $\mathrm{KAl} 60 \mathrm{ml}+\mathrm{CEF}$ & CEF & $21 d \times 3$ & (1) (5) \\
\hline Wang and Wang (2011) & $20 / 20$ & 46 & & & & & KAl $40 \mathrm{ml}+\mathrm{CTF}$ & CTF & $21 d \times 2$ & (2) (5) \\
\hline Zhu and Sun (2012) & $30 / 30$ & $\begin{array}{l}E: 52 \pm 5 \\
C: 54 \pm 3\end{array}$ & 1 & $17 / 19$ & $11 / 13$ & 1 & $\mathrm{KAl} 40 \mathrm{ml}+\mathrm{CAF}$ & CAF & $21 d \times 4$ & (1) \\
\hline Pan et al. (2016) & $45 / 45$ & $\begin{array}{l}\text { E: } 45.66 \pm 7.92 \\
\text { C: } 46.83 \pm 7.85\end{array}$ & / & / & $25 / 26$ & 20/19 & $\mathrm{KLTI} 200 \mathrm{ml}+\mathrm{CAF}$ & CAF & $21 d \times 6$ & (3) (4) (6) \\
\hline Fu et al. (2015) & $60 / 60$ & $\begin{array}{l}\text { E: } 46.7 \pm 7.8 \\
\text { C: } 47.5 \pm 7.1\end{array}$ & \multicolumn{4}{|c|}{ NR } & $\mathrm{KLTI} 200 \mathrm{ml}+\mathrm{CAF}$ & CAF & $21 d \times 6$ & (1) (4) (6) \\
\hline Yin et al. (1999) & $15 / 17$ & 52 & 3 & 13 & 16 & 1 & $\mathrm{KLTl} 200 \mathrm{ml}+\mathrm{FACT}$ & FACT & $21 d \times 2$ & (1) (2) (5) \\
\hline Gan et al. (2009) & $37 / 40$ & $\begin{array}{l}\text { E: } 45 \\
\text { C: } 46\end{array}$ & $16 / 18$ & $21 / 22$ & 1 & / & $\mathrm{KLTl} 100 \mathrm{ml}+\mathrm{CEF}$ & CEF & $21 d \times 2$ & (1) (2) (5) \\
\hline Jiang et al. (2017) & $55 / 55$ & $\begin{array}{l}\text { E: } 33-66 \\
C: 35-66\end{array}$ & / & / & $30 / 31$ & $25 / 24$ & $\mathrm{KLTI} 200 \mathrm{ml}+\mathrm{CAF}$ & CAF & $21 d \times 6$ & (6) \\
\hline Chen (2005) & $45 / 43$ & $\begin{array}{l}\text { E: } 35-61 \\
C: 35-59\end{array}$ & \multicolumn{4}{|c|}{ NR } & $\mathrm{SI} 40 \mathrm{ml}+\mathrm{CAF}$ & CAF & $21 d$ & (1) \\
\hline
\end{tabular}

Note: E, Treatment group; C, Control group; NR, Not reported; SQFZI, Shenqi Fuzheng injection; CKI, Compound Kushen injection; SMI, Shenmai injection; KAI, Kangai injection; ADI, Aidi injection; KLTI, Kanglaite injection; HQI, Huangqi injection; HCSI, Huachansu injection; SI, Shengmai injection; C, cyclophosphamide; F, 5-fluorouracil; A, doxorubicin; T, pirarubicin; E, epirubicin; M, methotrexate; (1): Clinical effectiveness rate; (2): Performance status; (3): T-lymphocyte subsets; (4): Peripheral hemogram; (5): ADRs; (): Tumor markers; (7): KPS; (8): ECG changes; (9): Comparison of left ventricular ejection fraction; (1): Liver and kidney function and myocardial enzyme spectrum. 


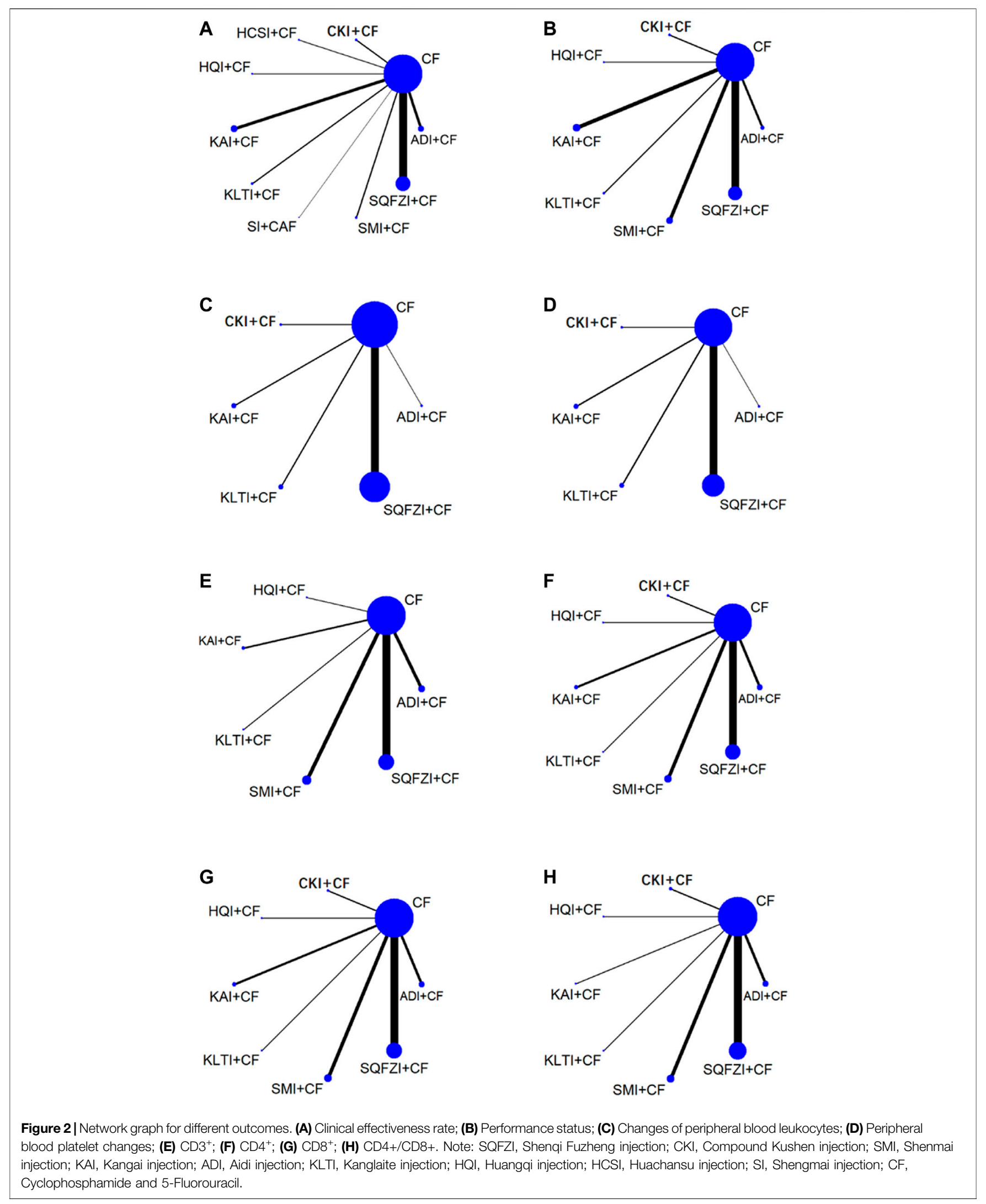




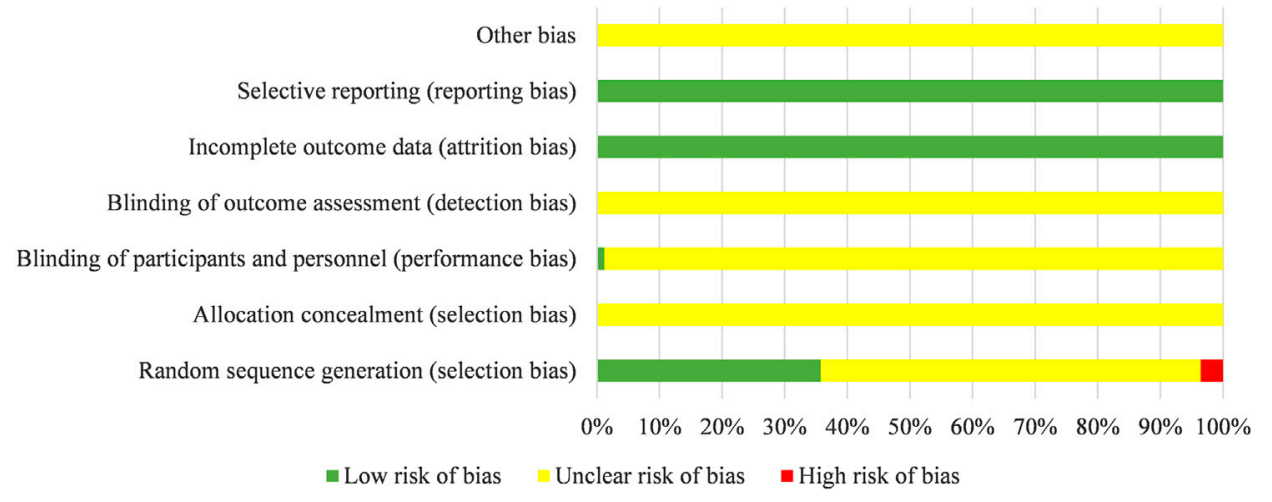

Figure $\mathbf{3}$ | Assessment of risk of bias.

\section{Peripheral Hemogram}

The report of peripheral hemogram included in this study mainly detected the changes of WBC and PLT. A total of 18 studies mentioned the changes of WBC, involving five CHIs and six interventions (ADI + CF, one RCT; SQFZI + CF, 12 RCTs; CKI + $\mathrm{CF}$, one RCT; KAI + CF, two RCTs; KLTI + CF, two RCTs). MDs showed that in improving WBC decline in $\mathrm{BC}$ patients, compared with CF alone, combining with ADI, SQFZI, CKI, KAI or KLTI on the basis of chemotherapy made no difference between groups. A total of 15 studies mentioned PLT, involving five CHIs and six interventions. Comparing to using $\mathrm{CF}$ alone, combined with ADI $(\mathrm{MD}=-36.03,95 \% \mathrm{CI}$ : $-71.43 \sim-1.37), \mathrm{KAI}(\mathrm{MD}=16.8,95 \% \mathrm{CI}$ : 9.17-24.62) on the basis of chemotherapy can improve PLT decline in patients and make the difference between groups statistically significant. Specific values are shown in Table 2. Meanwhile, the combination of $\mathrm{ADI}$ and $\mathrm{CF}$ compared with the combination of CKI and CF (MD $=-70.1$, 95\%CIs: $-130.90 \sim-0.81$ ), with the combination of KAI and CF $(\mathrm{MD}=$ -52.04 , 95\%CIs: $-88.72 \sim-17.52)$, the latter two were more effective in improving PLT decline in BC patients and the difference between groups were statistically significant.

After the ranking of each intervention efficacy, the combination of CF and CKI (61.9\%) had the highest probability of being the best treatment for $\mathrm{BC}$ in terms of improving WBC decline, followed by the combination of $\mathrm{CF}$ and SQFZI (56.6\%). The combination of CF and CKI (84\%) had the highest probability of being the best treatment for $\mathrm{BC}$ in terms of improving PLT decline, followed by the combination of CF and KAI (81.1\%) and the combination of CF and SQFZI (58.4\%). The ranking results of intervention measures are shown in Figure 4, and the SUCRA values are shown in Table 3.

\section{T-Lymphocyte Subsets}

The report of T-lymphocyte subsets in this study mainly involved four aspects: CD3+, CD4+, CD8+ and CD4+/CD8+. A total of 22 studies referred to $\mathrm{CD} 3+$, invoving six $\mathrm{CHI}$ and seven interventions (ADI + CF, four RCTs; SMI + CF, five RCTs; SQFZI + CF, nine RCTs, HQI + CF, one RCT; KAI + CF, two RCTs; KLTI + CF, one RCT). A total of 22 studies mentioned
$\mathrm{CD} 4+$ and $\mathrm{CD} 8+$, invoving seven $\mathrm{CHI}$ and eight interventions $(\mathrm{ADI}+\mathrm{CF}$, four RCTs; SMI + CF, five RCTs; SQFZI + CF, 11 RCTs; CKI + CF, two RCTs; HQI + CF, one RCT; KAI + CF, three RCTs; KLTI + CF, one RCT). A total of 22 studies mentioned $\mathrm{CD} 4+/ \mathrm{CD} 8+$, invoving seven CHIs and eight interventions (ADI + CF, four RCTs; SMI + CF, five RCTs; SQFZI + CF, 11 RCTs; $\mathrm{CKI}+\mathrm{CF}$, two RCTs; HQI + CF, one RCT; KAI + CF, one RCT; $\mathrm{KLTI}+\mathrm{CF}$, one RCT). MD values showed that, comparing to using CF alone, combined with SQFZI (MD = 10.41, 95\%CIs: 4.02-17.07) and KAI (MD = 20.34, 95\%CIs: 10.66-32.26) on the basis of chemotherapy can improve the decline of immune function of $\mathrm{CD} 3+$ in $\mathrm{BC}$ patients and make the difference between groups statistically significant. Combined with SQFZI $(\mathrm{MD}=6.91,95 \% \mathrm{CIs}: 2.03-11.94)$ and CKI (MD = 10.26, 95\%CIs: 3.18-17.41) on the basis of chemotherapy can improve the decline of $\mathrm{CD} 4+$ and $\mathrm{CD} 8+$ in $\mathrm{BC}$ patients and make the difference between groups statistically significant. Combined with SQFZI (MD $=0.54$, 95\%CIs: $0.06-0.97)$ on the basis of chemotherapy can improve the decline of $\mathrm{CD} 4+/ \mathrm{CD} 8+$ in $\mathrm{BC}$ patients and make the difference between groups statistically significant. Specific values are shown in Table 2 . Above outcomes showed that $\mathrm{CF}$ alone can inhibit the body's immune function, while the combination of $\mathrm{CF}$ and $\mathrm{CHIs}$ can reduce the inhibition of immune function caused by CF.

After the ranking of each intervention efficacy, the combination of CF and KAI (94.3\%) had the highest probability of being the best treatment for $\mathrm{BC}$ in the respect of reducing the decline of $\mathrm{CD} 3+$, followed by the combination of CF and SQFZI (70.2\%) and the combination of CF and KLTI (58.9\%). The combination of CF and CKI (83.7\%) had the highest probability of being the best treatment for $\mathrm{BC}$ in the respect of reducing the decline of CD4+ and CD8+, followed by the combination of CF and KAI (69.8\%) and the combination of CF and SQFZI (68.4\%). The combination of CF and SQFZI (72.1\%) had the highest probability of being the best treatment for $\mathrm{BC}$ in the respect of reducing the decline of $\mathrm{CD} 4+/ \mathrm{CD} 8+$, followed by the combination of $\mathrm{CF}$ and KAI (58.5\%) and the combination of $\mathrm{CF}$ and $\mathrm{ADI}$ (57.8\%). The ranking results of interventions are shown in Figure 4, and the SUCRA values are shown in Table 3. 
TABLE 2 | Statistical results of network meta-analysis for the main outcomes (OR/MD value, 95\% Cl).

$\begin{array}{ccccccc}\begin{array}{c}\text { Clinical } \\ \text { effectiveness } \\ \text { rate }^{\mathrm{a}}\end{array} & \begin{array}{c}\text { Performance } \\ \text { status }^{\mathrm{a}}\end{array} & \text { WBC } & \text { PLT } & \text { CD3+ } & \text { CD4+ } & \text { CD8+ } \\ & & & & \end{array}$

$0.61(0,29,178)$
$\mathrm{SMl}+\mathrm{CF}$ $\mathrm{SQFZI}+\mathrm{CF}$

$\mathrm{CKI}+\mathrm{CF}$

$\mathrm{HCSI}+\mathrm{CF}$

$\mathrm{HQI}+\mathrm{CF}$

$\mathrm{KAl}+\mathrm{CF}$

$\mathrm{KLTI}+\mathrm{CF}$

$\mathrm{SI}+\mathrm{CF}$

$\mathrm{CF}$

$\mathrm{SMI}+\mathrm{CF}$ vs

$\mathrm{SQFZI}+\mathrm{CF}$

$\mathrm{CKI}+\mathrm{CF}$

$\mathrm{HCSI}+\mathrm{CF}$

$\mathrm{HQI}+\mathrm{CF}$

$\mathrm{KAl}+\mathrm{CF}$

$\mathrm{KLTI}+\mathrm{CF}$

$\mathrm{SI}+\mathrm{CF}$

CF

\section{SQFZI + CF vs}

$\mathrm{CKI}+\mathrm{CF}$

$\mathrm{HCSI}+\mathrm{CF}$

$\mathrm{HQI}+\mathrm{CF}$

$\mathrm{KAl}+\mathrm{CF}$

$\mathrm{KLTI}+\mathrm{CF}$

$\mathrm{SI}+\mathrm{CF}$

CF

CKI + CF vs

$\mathrm{HCSI}+\mathrm{CF}$

$\mathrm{HQI}+\mathrm{CF}$

$\mathrm{KAl}+\mathrm{CF}$

$\mathrm{KLTI}+\mathrm{CF}$

$\mathrm{SI}+\mathrm{CF}$

CF

\section{HCSI + CF vs}

$\mathrm{HQI}+\mathrm{CF}$

$\mathrm{KAl}+\mathrm{CF}$

$\mathrm{KLTI}+\mathrm{CF}$

$\mathrm{SI}+\mathrm{CF}$

CF

HQI + CF vs

$\mathrm{KAl}+\mathrm{CF}$

$\mathrm{KLTI}+\mathrm{CF}$

$\mathrm{SI}+\mathrm{CF}$

CF

$0.83(0.45,1.99)$

$0.98(0.62,1.88)$

$0.79(0.34,2.02)$

$0.84(0.41,1.81)$

$0.66(0.31,1.77)$

$0.95(0.58,1.91)$

$1.53(0.67,3.61)$

$2.45(0.80,14.95)$

$\underline{0.43(0.30,0.75)}$

$1.06(0.62,2.28)$

$0.9(0.39,2.38)$

$1.02(0.37,2.21)$

$0.78(0.29,1.98)$

$1.12(0.56,2.36)$

$1.85(0.66,4.32)$

$2.9(0.84,16.52)$

$\underline{0.47(0.27,0.93)}$

$0.86(0.40,1.60)$

$0.91(0.38,1.53)$

$0.75(0.30,1.46)$

$1.03(0.62,1.58)$

$1.61(0.72,2.98)$

$2.45(0.88,14.72)$

$0.45(0.33,0.55)$

$1.09(0.40,2.55)$

$0.86(0.30,2.40)$

$1.25(0.56,2.57)$

$1.99(0.72,4.83)$

$2.81(0.95,24.22)$

$0.52(0.27,1.04)$

$0.77(0.32,2.35)$

$1.08(0.60,2.63)$

$1.79(0.76,4.86)$

$3.01(0.90,18.46)$

$0.49(0.29,1.05)$

$1.42(0.63,3.33)$

$2.29(0.83,6.05)$

3.65 (1.02, 23.06)
$0.61(0.29,1.78)$

$0.72(0.30,1.79)$

$0.42(0.18,1.58)$

$0.59(0.17,2.96)$

$0.68(0.24,1.99)$

$0.77(0.17,3.04)$

$0.21(0.09,0.47)$

$1.15(0.63,2.07)$

$0.71(0.31,1.97)$

$1.00(0.29,4.29)$

$1.04(0.52,2.56)$

$1.17(0.39,4.28)$

$0.32(0.20,0.55)$

$0.61(0.28,1.49)$

$0.88(0.25,3.39)$

$0.91(0.50,1.97)$

$1.06(0.31,3.06)$

$0.29(0.20,0.39)$

$1.42(0.34,5.75)$

$1.51(0.58,3.80)$

$1.7(0.51,5.49)$

$0.45(0.20,0.95)$

$-0.77(-5.09,3.38)$

$-39.97(-92.09,2.38)$

$-70.1(-130.90,-0.81)$

$$
-
$$

$-0.36(-7.97,6.19)$

$-0.62(-4.63,3.82)$

$-0.61(-4.28,3.57)$

-
-
-
-
-
-
-
-

$-0.64(-4.66,4.92)$

$$
-
$$

$0.55(-4.24,4.82)$

$0.14(-1.24,1.64)$

$0.1(-0.72,1.20)$

$$
-
$$

$1.01(-5.63,6.79)$ $0.76(-5.04,4.90)$

$1.11(0.23,4.27)$

$1.09(0.29,5.64)$

$0.33(0.09,1.06)$

$\frac{52.04\left(-\frac{88.72,}{-9.90}(-62.70,29.80)\right.}{-9.52)}$

-36.03 (-71.43, $-\underline{-1.37)}$

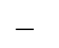

$-$

$-$

$-$

$-$

$-31.32(-81.55,44.85)$

$-12.51(-40.08,11.11)$

$28.07(-25.15,86.27)$

$2.79(-21.01,24.86)$

$14.43(-68.54,71.25)$

$58.15(-1.22,121.40)$

$0.73(-4.90,4.77)$

$31.67(-51.02,86.36)$
$15.78(-25.26,-6.78)$

$-3.62(-13.24,7.48)$

$-8.45(-16.90,0.77)$

$-11.7(-21.27,-1.38)$

$-$

$-8.95(-19.29,2.64)$

$-9.18(-38.32,21.07)$

$3.97(-50.11,15.71)$

$-1.43(-8.54,6.36)$

$-4.72(-15.36,5.33)$$$
-
$$

$4.91(-12.64,24.80)$

$14.43(-29.33,-1.55)$

$-2.65(-38.50,26.13)$

$5.53(-2.58,13.63)$

$1.41(-32.78,31.08)$

$10.41(4.02,17.07)$
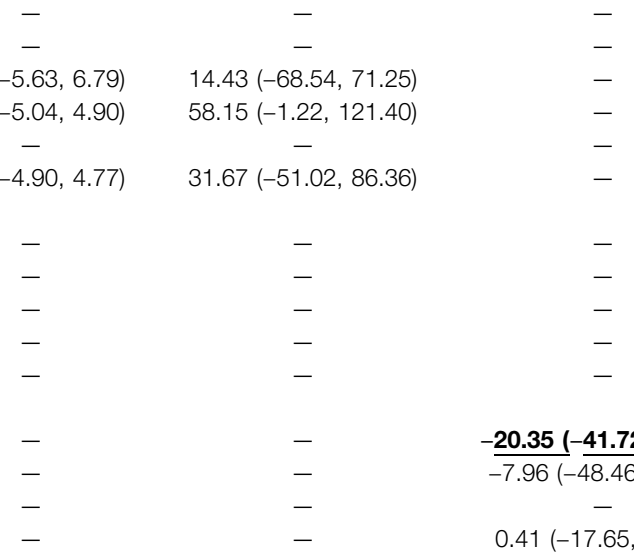

$2.16(-14.55,18.14)$

$-5.31(-16.26,5.06)$

$-5.50(-34.17,23.61)$

$-$

$-3.39(-11.96,5.47)$

$6.87(-9.52,23.22)$

$-0.43(-9.92,8.63)$

$-0.60(-30.01,28.50)$

$6.91(2.03,11.94)$

$10.32(-7.29,27.01)$

$2.92(-7.64,13.19)$

$2.58(-27.23,32.86)$

$10.26(3.18,17.41)$
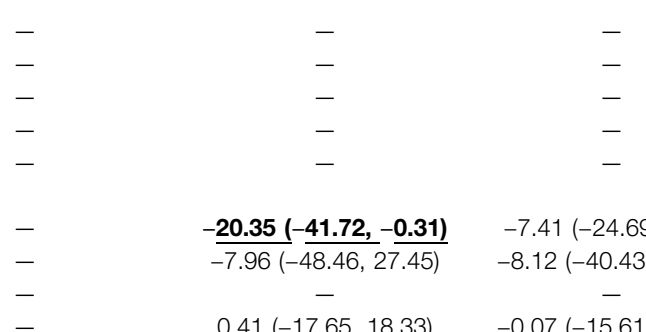

$-$

$\frac{\mathbf{2 0 . 3 5}(-\mathbf{4 1 . 7 2},-\mathbf{0 . 3 1})}{-7.96(-48.46,27.45)}$

$0.41(-17.65,18.33)$ $-8.12(-40.43,25.51)$
$-0.07(-15.61,15.98)$
$-5.38(-24.93,13.34)$

-

$-12.51(-40.08,11.11)$

$-3.62(-13.24,7.48) \quad 0.18(-0.96,1.35)$

$-8.44(-16.90,0.77) \quad-0.14(-1.09,0.95)$

$\mathbf{- 1 1 . 7}(-\mathbf{2 1 . 2 7}, \mathbf{- 1 . 3 8}) \quad 0.21(-0.91,1.24)$

$-1.29(-18.56,15.91) \quad 0.18(-1.66,1.94)$

$-8.95(-19.29,2.64) \quad 0.03(-1.23,1.11)$

$-9.18(-38.32,21.07) \quad 0.07(-2.47,2.52)$

$-1.43(-8.54,6.36) \quad 0.42(-0.52,1.35)$

$-4.85(-13.72,3.95) \quad-4.85(-13.72,3.95) \quad-0.31(-0.98,0.45)$

$-8.30(-18.26,2.07) \quad-8.30(-18.26,2.07) \quad 0.02(-0.81,0.77)$

$2.16(-14.55,18.14)$

$-5.31(-16.26,5.06)$

$-5.50(-34.17,23.61)$

$0.01(-1.67,1.46)$

$-0.16(-1.10,0.71)$

$-0.20(-2.24,2.20)$

$2.14(-5.45,9.31) \quad 0.24(-0.30,0.79)$

$-3.39(-11.96,5.47)$

$6.87(-9.52,23.22)$

$-0.44(-9.92,8.63)$

$-0.60(-30.01,28.50)$

$0.32(-0.49,0.97)$

$0.34(-1.30,1.64)$

$0.15(-0.77,0.95)$

$0.12(-1.93,2.65)$

$6.91(2.03,11.94)$

$\underline{0.54(0.06,0.97)}$

$10.32(-7.29,27.01)$

$2.92(-7.64,13.19)$

$2.58(-27.23,32.86)$

$-0.01(-1.56,1.32)$

$-0.18(-1.08,0.79)$

$-0.17(-2.22,2.17)$

$10.26(3.18,17.41)$

$0.21(-0.28,0.81)$

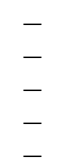

$-7.41(-24.69,9.68) \quad-0.16(-1.60,1.50)$ $-8.12(-40.43,25.51) \quad-0.14(-2.45,2.31)$

$-0.07(-15.61,15.98) \quad 0.25(-1.05,1.74)$

(Continued on following page) 


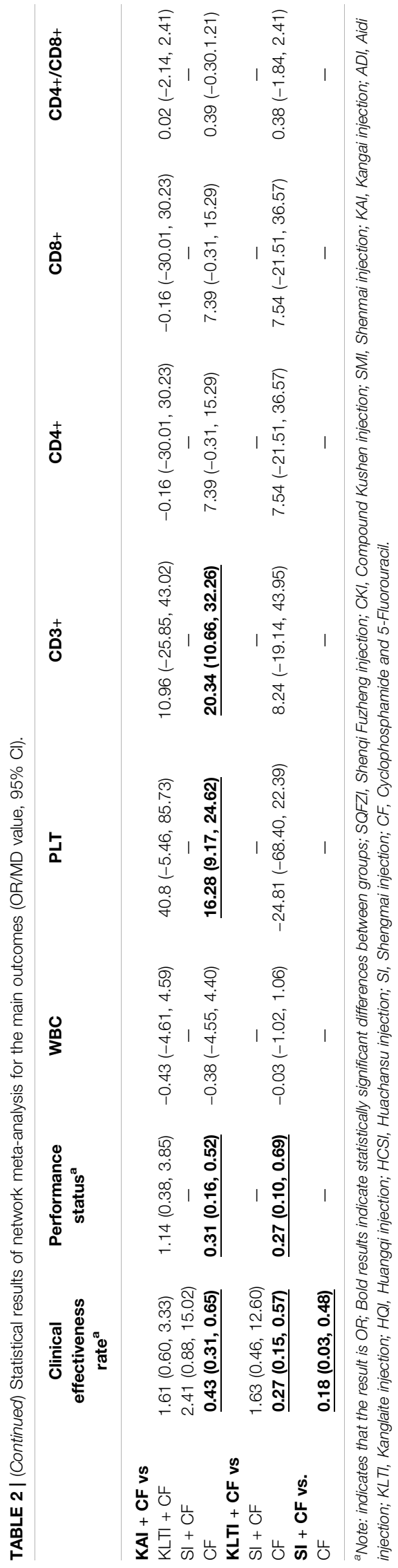

\section{Adverse Reactions}

Only one study clearly reported that no ADRs occurred during the study, and 53 studies had ADRs during the study. The ADRs mainly included five aspects: gastrointestinal reaction, abnormal renal and liver function, hair loss, nausea and vomiting, and decreased WBC.

A total of 12 studies reported gastrointestinal reactions, involving four kinds of CHIs. The result of NMA showed that CF combined with ADI (OR $=2.17,95 \%$ CIs: $1.03-4.79)$, SQFZI $(\mathrm{OR}=5.32,95 \%$ CIs: $2.50-9.50)$, and $\mathrm{KAI}(\mathrm{OR}=2.34,95 \% \mathrm{CIs}$ : 1.24-4.79) can make statistically significant difference compared with using CF alone. SQFZI combined with CF was better than HCSI combined with CF (OR $=7.43$, 95\%CIs: $1.19-34.61)$ in the respect of relieving gastrointestinal reactions (Table 4).

A total of 19 studies reported abnormal renal and liver function, involving five kinds of CHIs. NMA showed that $\mathrm{CF}$ combined with SQFZI (OR $=3.89,95 \%$ CIs: $1.94-7.90)$, CKI (OR $=3.52$, 95\%CIs: $1.27-12.66)$, and KAI $(\mathrm{OR}=3.16,95 \%$ CIs: 1.38-9.00) can make statistically significant difference compared with using CF alone (Table 4).

A total of 13 studies reported hair loss, involving five kinds of CHIs. NMA showed that CF combined with $\mathrm{KAI}(\mathrm{OR}=3.97,95 \%$ CIs: 1.19-15.89) can make statistically significant difference compared with using CF alone (Table 4).

A total of 21 studies reported decreased WBC, involving five kinds of CHIs. NMA showed that CF combined with SMI (OR = 4.15, 95\%CIs: $2.28-7.32)$, SQFZI (OR = 2.49, 95\%CIs: 1.57-4.41), HQI (OR $=3.49$, 95\%CIs: $1.01-11.48)$, and KAI (OR $=2.80,95 \%$ CIs: $1.67-5.19)$ can make statistically significant difference compared with using $\mathrm{CF}$ alone (Table 4).

A total of 19 studies reported nausea and vomiting, involving seven kinds of CHIs. NMA showed that, $\mathrm{CF}$ combined with ADI $(\mathrm{OR}=6.84,95 \%$ CIs: $1.73-51.08)$, SMI $(\mathrm{OR}=3.74,95 \%$ CIs: 2.18-7.77), SQFZI (OR $=3.31$, 95\%CIs: $1.87-6.03)$, CKI $(\mathrm{OR}=2.86$, 95\%CIs: $1.27-6.11)$, HQI $(\mathrm{OR}=3.84,95 \%$ CIs: 1.45-10.60), and KAI (OR = 2.57, 95\%CIs: 1.10-6.10) can make statistically significant difference compared with using CF alone (Table 4).

The results of area under the adverse reaction curve showed that, SQFZI combined with CF had the highest likelihood of being the best treatment for improving the gastrointestinal reaction (96.7\%) and abnormal renal and liver function (77.8\%) in BC patients, KAI combined with CF had a better effect on the symptoms of hair loss (73.5\%), SMI had more advantages in reducing decreased WBC (80.9\%), and ADI combined with CF was more effective in reducing nausea and vomiting $(82.8 \%)$. The details are shown in Table 5.

In 53 studies that reported ADRs, interventions of the control group were all $\mathrm{CF}$, and other ADRs involved were reported as follows. ADI combined with $\mathrm{CF}$ was used as a treatment group intervention in seven RCTs: In Song's study (Song et al., 2006), 21 patients in the treatment group had myelosuppression of more than a degree, while 24 in the control group. In Chen's study (Chen W. M., 2016), there were 8 cases of bone marrow suppression in the treatment group and 18 cases in the control group. In Dang's study (Dang and Wang, 2010), there was 1 case of III, IV cardiac function changes in the treatment group and 3 cases in the control group. In 
A

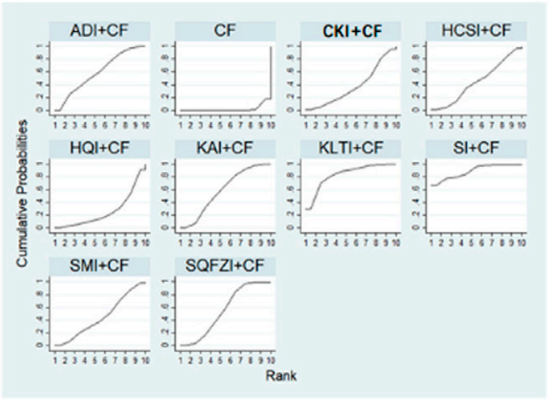

C

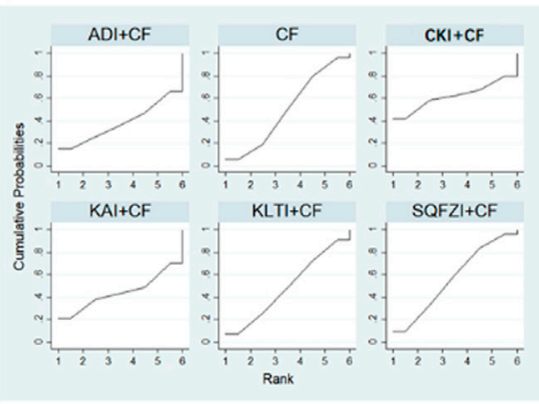

E

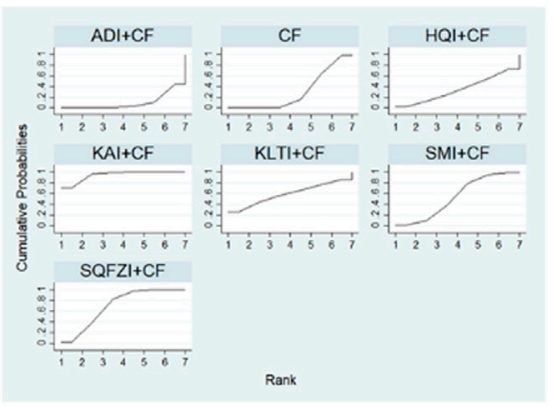

G

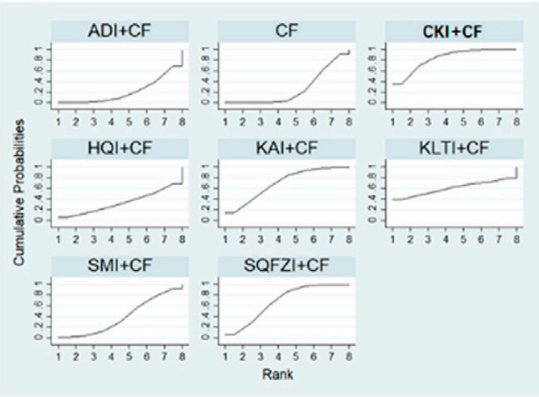

B

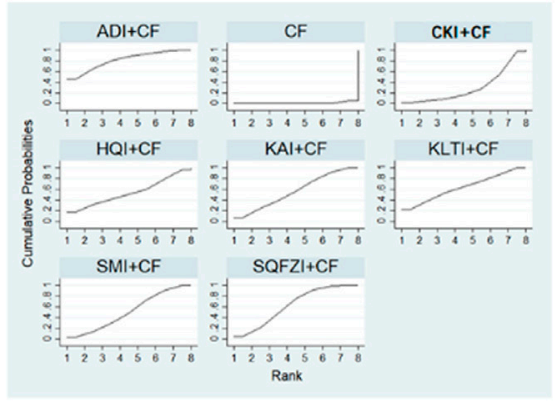

D

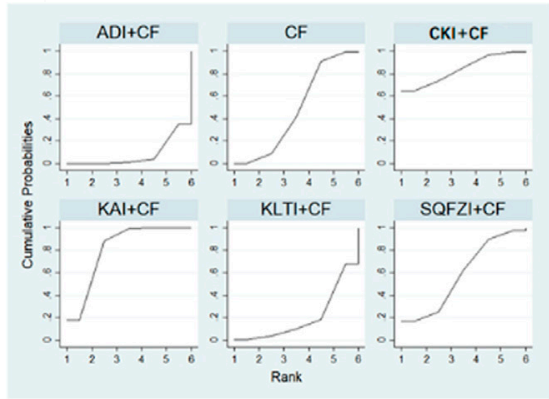

F

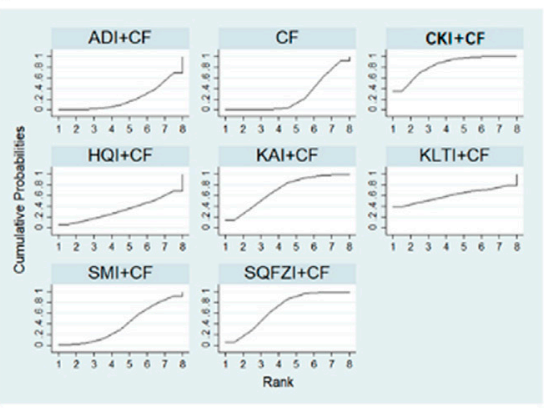

H

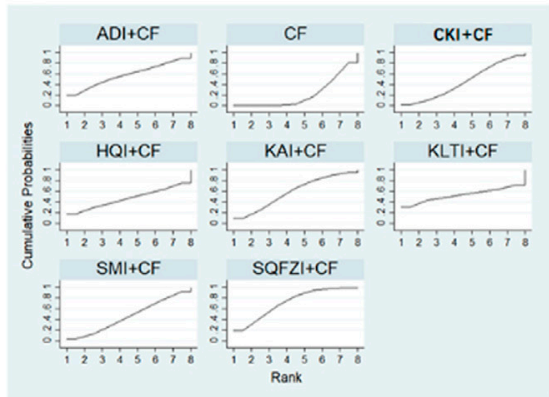

Figure 4 | Plot of the surface under the cumulative ranking curves for all treatments. (A) Clinical effectiveness rate; (B) Performance status; (C) Changes of peripheral blood leukocytes; (D) Peripheral blood platelet changes; (E) $\mathrm{CD}^{+}$; (F) $\mathrm{CD}^{+}$; (G) $\mathrm{CD}^{+}$; (H) $\mathrm{CD} 4+/ \mathrm{CD} 8+$. Note: SQFZl, Shenqi Fuzheng injection; CKI, Compound Kushen injection; SMI, Shenmai injection; KAl, Kangai injection; ADI, Aidi injection; KLTI, Kanglaite injection; HQI, Huangqi injection; HCSI, Huachansu injection; SI, Shengmai injection; CF, Cyclophosphamide and 5-Fluorouracil. 

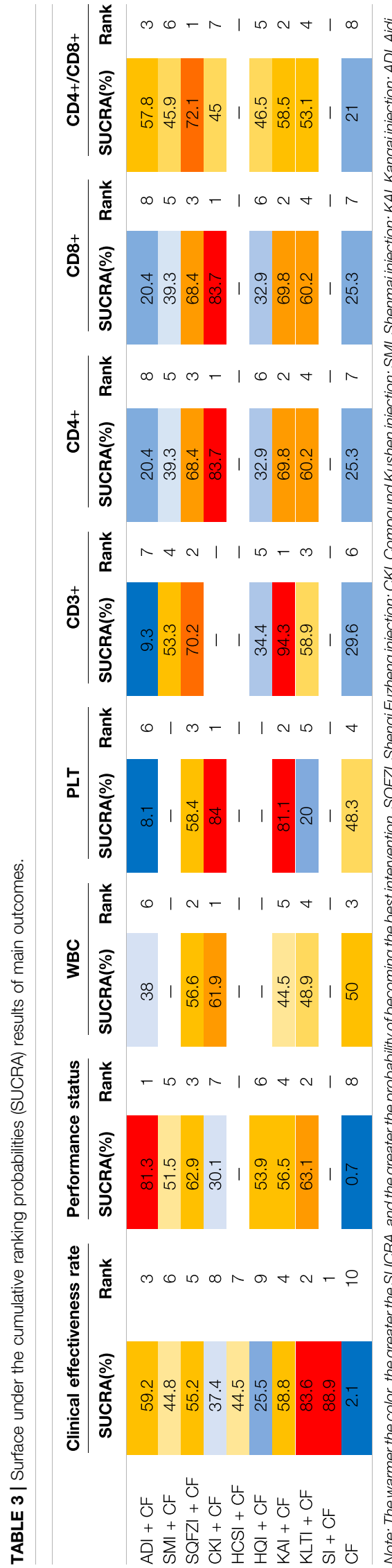

Liu's study (Liu et al., 2005), there were only 2 cases of decreased WBC in the control group. The blood image was reduced in 30 cases in the treatment group and 40 cases in the control group, they had statistically significant difference $(p<0.05)$. In the treatment group, individual patients presented with fever, urticaria and other symptoms, which were relieved after symptomatic treatment. In Ye's study (Ye et al., 2002), there were 8 cases of mild drug fever less than $38.5^{\circ} \mathrm{C}$. In Ren's study (Ren and Zhang, 2005), the main side effects were nausea and vomiting grade I and II, and decreased WBC. The peripheral hemogram in the treatment group was not significantly reduced, but individual patients developed the symptoms of fever and urticaria. The peripheral hemogram of the control group decreased significantly. In Han's study (Han et al., 2014), there were 15 cases of myelosuppression in the treatment group and 32 cases in the control group.

SMI combined with CF was used as a treatment group intervention in nine RCTs: In Xiong's study (Xiong et al., 2006), there were 12 cases of ST-T change, 2 cases of QT-QTc extension, 4 cases of sinus tachycardia, 2 cases of QRS low voltage, 3 cases of artrial premature beat, and 1 case of ventricular premature beat in the treatment group, and the incidence was $55.8 \%$. The above symptoms in the control group were 3 cases, 0 cases, 1 case, 1 case, 2 cases, 0 cases, and the incidence was $17.5 \%$. The incidence of electrocardiographic abnormality in the treatment group was significantly higher than that in the control group $(p<0.01)$. In Wang's study (Wang et al., 2012), there were 4 cases of ST-T changes, 3 cases of QT internal prolongation, 1 case of QRS low voltage, 5 cases of nodal tachycardia, 4 cases of premature ventricular beats, 3 cases of ventricular premature contraction, and 2 cases of atrial fibrillation in the treatment group. While in the control group, there were 17 cases, 10 cases, 2 cases, 13 cases, 12 cases, 7 cases, 7 cases respectively. The incidence rates of ST-T changes, QT internal prolongation, nodal tachycardia, premature ventricular beats, and ventricular premature contraction in the treatment group were all lower than that of the control group, their difference was statistically significant $(p<0.05$ or $p<0.01$ ). There was no statistically significant difference between the two groups in the incidence of QRS low voltage and atrial fibrillation $(p>0.05)$. In Yao's study (Yao et al., 2016), both treatment group and control group had the symptoms of shortness of breath, fatigue, irritability, hot flashes, night sweats, dry mouth and dry throat. However, the scores of TCM symptoms in the treatment group were significantly lower than those in the control group. In Chen's study (Chen et al., 2018), the adverse reactions of weak, spontaneous sweating, dry mouth and nervous exhaustion were reported. The clinical symptom scores of both groups were lower than those before treatment, and the treatment group was lower than the control group. Zhang's study (Zhang, 2013) reported bone marrow suppresses gastrointestinal reactions, hair loss, nausea and vomiting, and decreased WBC. In Yang's study (Yang, 2008), 105 patients in the control group had palpitation, chest distress, nocturnal paroxysmal dyspnea and other discomfort, and one of them had paroxysmal syncope. Only 61 patients in the treatment group had the discomfort of palpitation and chest distress. There were 155 cases of abnormal electrocardiogram in the control group and 109 
TABLE 4 | Statistical results of network meta-analysis for ADR outcomes (OR value, 95\% Cl).

\begin{tabular}{|c|c|c|c|c|c|}
\hline & $\begin{array}{l}\text { Gastrointestinal } \\
\text { reactions }\end{array}$ & $\begin{array}{l}\text { Abnormal renal } \\
\text { and liver } \\
\text { function }\end{array}$ & Hair loss & Decreased WBC & $\begin{array}{l}\text { Nausea and } \\
\text { vomiting }\end{array}$ \\
\hline \multicolumn{6}{|l|}{$A D I+C F$ vs } \\
\hline $\mathrm{SMl}+\mathrm{CF}$ & - & - & $0.40(0.02,6.29)$ & - & $1.74(0.39,12.74)$ \\
\hline SQFZI + CF & $0.42(0.16,1.26)$ & $0.36(0.15,1.07)$ & $0.54(0.02,8.44)$ & $1.63(0.67,3.57)$ & $2.19(0.41,15.25)$ \\
\hline $\mathrm{CKI}+\mathrm{CF}$ & - & $0.37(0.10,1.50)$ & - & $1.50(0.43,6.13)$ & $2.51(0.48,19.13)$ \\
\hline $\mathrm{HCSI}+\mathrm{CF}$ & $2.99(0.47,16.06)$ & $0.82(0.05,7.03)$ & - & - & \\
\hline $\mathrm{HQI}+\mathrm{CF}$ & - & - & $0.60(0.01,24.53)$ & $1.22(0.29,3.53)$ & $1.95(0.33,15.80)$ \\
\hline $\mathrm{KAl}+\mathrm{CF}$ & $0.92(0.33,2.66)$ & $0.45(0.14,1.63)$ & $0.35(0.02,6.00)$ & $1.48(0.67,3.35)$ & $2.87(0.46,24.95)$ \\
\hline $\mathrm{KLTI}+\mathrm{CF}$ & & & & & $3.62(0.43,34.55)$ \\
\hline CF & $2.17(1.03,4.79)$ & $1.40(0.71,3.46)$ & $1.43(0.11,17.83)$ & $4.15(2.28,7.32)$ & $6.84(1.73,51.08)$ \\
\hline \multicolumn{6}{|l|}{ SMI + CF vs } \\
\hline SQFZI + CF & - & - & $1.38(0.13,9.24)$ & - & $1.14(0.48,2.89)$ \\
\hline $\mathrm{CKI}+\mathrm{CF}$ & - & - & - & - & $1.35(0.51,3.74)$ \\
\hline $\mathrm{HCSI}+\mathrm{CF}$ & - & - & - & - & - \\
\hline $\mathrm{HQI}+\mathrm{CF}$ & - & - & $1.55(0.08,27.36)$ & - & $0.98(0.31,3.10)$ \\
\hline $\mathrm{KAl}+\mathrm{CF}$ & - & - & $0.91(0.12,5.39)$ & - & $1.47(0.51,4.34)$ \\
\hline $\mathrm{KLTI}+\mathrm{CF}$ & - & - & - & - & $1.89(0.41,8.92)$ \\
\hline CF & - & - & $3.62(0.99,14.46)$ & - & $3.74(2.18,7.77)$ \\
\hline \multicolumn{6}{|l|}{ SQFZI + CF vs } \\
\hline $\mathrm{CKI}+\mathrm{CF}$ & - & $1.15(0.21,3.76)$ & - & $0.94(0.26,3.72)$ & $1.17(0.45,3.73)$ \\
\hline $\mathrm{HCSI}+\mathrm{CF}$ & $7.43(1.19,34.61)$ & $2.11(0.14,15.74)$ & - & - & - \\
\hline $\mathrm{HQI}+\mathrm{CF}$ & - & - & $1.15(0.06,29.40)$ & $0.71(0.20,2.35)$ & $0.86(0.26,2.83)$ \\
\hline $\mathrm{KAl}+\mathrm{CF}$ & $2.20(0.76,5.19)$ & $1.21(0.37,3.24)$ & $0.67(0.09,6.15)$ & $0.90(0.42,1.95)$ & $1.31(0.44,3.48)$ \\
\hline $\mathrm{KLTI}+\mathrm{CF}$ & - & - & - & - & $1.65(0.37,7.72)$ \\
\hline CF & $5.32(2.50,9.50)$ & $3.89(1.94,7.90)$ & $2.66(0.61,17.08)$ & $2.49(1.57,4.41)$ & $3.31(1.87,6.03)$ \\
\hline \multicolumn{6}{|l|}{ CKI + CF vs } \\
\hline $\mathrm{HCSI}+\mathrm{CF}$ & - & $2.07(0.12,18.66)$ & - & - & - \\
\hline $\mathrm{HQI}+\mathrm{CF}$ & - & - & - & 0.77 (0.12, 3.83) & $0.74(0.20,2.51)$ \\
\hline $\mathrm{KAl}+\mathrm{CF}$ & - & $1.18(0.24,5.36)$ & - & $0.99(0.22,3.55)$ & $1.10(0.33,3.61)$ \\
\hline $\mathrm{KLTI}+\mathrm{CF}$ & - & - & - & - & $1.37(0.29,6.84)$ \\
\hline CF & - & $3.52(1.27,12.66)$ & - & $2.74(0.71,9.13)$ & $2.86(1.27,6.11)$ \\
\hline \multicolumn{6}{|l|}{ HCSI + CF vs } \\
\hline $\mathrm{HQI}+\mathrm{CF}$ & - & - & - & - & - \\
\hline $\mathrm{KAl}+\mathrm{CF}$ & $0.30(0.06,1.88)$ & $0.59(0.07,7.61)$ & - & - & - \\
\hline CF & $0.69(0.17,3.76)$ & $1.73(0.28,25.26)$ & - & - & - \\
\hline \multicolumn{6}{|l|}{ HQI + CF vs } \\
\hline $\mathrm{KAl}+\mathrm{CF}$ & - & - & $0.60(0.03,10.65)$ & $1.26(0.34,4.10)$ & $1.53(0.38,6.12)$ \\
\hline $\mathrm{KLTI}+\mathrm{CF}$ & - & - & - & - & $1.91(0.34,11.86)$ \\
\hline CF & - & - & $2.36(0.19,34.91)$ & $3.49(1.01,11.48)$ & $3.84(1.45,10.60)$ \\
\hline \multicolumn{6}{|l|}{ KAl + CF vs } \\
\hline $\mathrm{KLTI}+\mathrm{CF}$ & - & - & - & - & $1.28(0.24,6.16)$ \\
\hline CF & $2.34(1.24,4.79)$ & $3.16(1.38,9.00)$ & $3.97(1.19,15.89)$ & $2.80(1.67,5.19)$ & $2.57(1.10,6.10)$ \\
\hline \multicolumn{6}{|l|}{ KLTI + CF vs } \\
\hline CF & - & - & - & - & $2.02(0.48,8.44)$ \\
\hline
\end{tabular}

Note: Bold results indicate statistically significant differences between groups; SQFZI, Shenqi Fuzheng injection; CKI, Compound Kushen injection; SMI, Shenmai injection; KAl, Kangai injection; ADI, Aidi injection; KLTI, Kanglaite injection; HQI, Huangqi injection; HCSI, Huachansu injection; SI, Shengmai injection; CF, Cyclophosphamide and 5-Fluorouracil.

cases in the treatment group. There were 182 cases of abnormal dynamic electrocardiogram in the control group and 134 cases in the treatment group.

SQFZI combined with CF was used as a treatment group intervention in 16 RCTs: In Liu's study (Liu, 2017), two patients in the treatment group had decreased hemoglobin. While in the control group, five patients had decreased hemoglobin, four patients with liver and kidney poisoning, and two patients had hair loss. The overall incidence of ADRs in treatment group was markedly lower than that in control group. In Zou's study (Zou et al., 2006), according to the ECOG physical condition score, 13, 12,6 , and 0 patients in the treatment group were at grade $0, \mathrm{I}, \mathrm{II}$ and III respectively. While there were 3 cases, 6 cases, 8 cases and 14 cases of grade 0 -III physical condition in the control group. The number of fatigue cases in the treatment group was dramatically lower than that in the control group $(p<0.05)$. Regarding cardiac toxicity, there were 3 cases in Grade I, and 10 cases in Grade II in treatment group. While there were 4 cases in Grade I, 10 cases in grade II, and 4 cases in Grade III in control group. The number of tachycardia, premature beats, or ST-T changes in electrocardiogram in treatment group was less than that in control group after the second chemotherapy. In Song's study (Song, 2004), there were 2 cases with WBC less than $3.0 \times$ $10^{9} \mathrm{~g} / \mathrm{L}$ after treatment, 1 case with hemoglobin less than $80 \mathrm{~g} / \mathrm{L}$, 
TABLE 5 | Surface under the cumulative ranking probabilities (SUCRA) results of ADR outcomes.

\begin{tabular}{|c|c|c|c|c|c|c|c|c|c|c|}
\hline & \multicolumn{2}{|c|}{$\begin{array}{l}\text { Gastrointestinal } \\
\text { reactions }\end{array}$} & \multicolumn{2}{|c|}{$\begin{array}{l}\text { Abnormal renal and } \\
\text { liver function }\end{array}$} & \multicolumn{2}{|c|}{ Hair loss } & \multicolumn{2}{|c|}{ Decreased WBC } & \multicolumn{2}{|c|}{ Nausea and vomiting } \\
\hline & SUCRA(\%) & Rank & SUCRA(\%) & Rank & SUCRA(\%) & Rank & SUCRA(\%) & Rank & SUCRA(\%) & Rank \\
\hline$A D I+C F$ & 59.10 & 3 & 29.20 & 5 & 35.00 & 5 & - & - & 82.8 & 1 \\
\hline $\mathrm{SMI}+\mathrm{CF}$ & - & - & - & - & 69.30 & 2 & 80.90 & 1 & 65.9 & 2 \\
\hline SQFZI + CF & 96.70 & 1 & 77.80 & 1 & 56.80 & 3 & 45.00 & 5 & 57.4 & 4 \\
\hline $\mathrm{CKI}+\mathrm{CF}$ & - & - & 73.20 & 2 & - & - & 52.10 & 4 & 48.2 & 5 \\
\hline $\mathrm{HCSI}+\mathrm{CF}$ & 13.50 & 5 & 44.70 & 4 & - & - & - & - & - & - \\
\hline $\mathrm{HQI}+\mathrm{CF}$ & - & - & - & - & 51.30 & 4 & 67.20 & 2 & 65.3 & 3 \\
\hline $\mathrm{KAl}+\mathrm{CF}$ & 63.30 & 2 & 66.70 & 3 & 73.50 & 1 & 53.00 & 3 & 43.1 & 6 \\
\hline $\mathrm{KLTI}+\mathrm{CF}$ & - & - & - & - & - & - & - & - & 34.9 & 7 \\
\hline CF & 17.30 & 4 & 8.40 & 6 & 14.20 & 6 & 1.80 & 6 & 2.5 & 8 \\
\hline
\end{tabular}

Note: The warmer the color, the greater the SUCRA, and the greater the probability of becoming the best intervention. SQFZI, Shenqi Fuzheng injection; CKI, Compound Kushen injection; SMI, Shenmai injection; KAl, Kangai injection; ADI, Aidi injection; KLTI, Kanglaite injection; HQI, Huangqi injection; HCSI, Huachansu injection; SI, Shengmai injection; CF,

Cyclophosphamide and 5-Fluorouracil.

and 0 cases with PLT less than $60 \times 10^{9} \mathrm{~g} / \mathrm{L}$. While in the control group, there were 8 cases, 3 cases, and 1 case respectively, and the difference was significant between two groups $(p<0.05)$. In Chen's study (Chen and Lin, 2007), ADRs were hemotoxicity, non-hemotoxicity and cardiotoxicity. The hemotoxicity was primarily the decrease of WBC, of which the decrease of treatment group was smaller than that of control group and the difference was statistically significant. There was no significant difference in the decrease of PLT and hemoglobin. Non-hemotoxicity reactions caused by chemotherapy drugs included nausea, vomiting, diarrhea, cardiotoxic, stomatitis, and hair loss. The number of fatigue, nausea and vomiting in treatment group was significantly lower than that in control group, and the difference was statistically significant $(p<$ $0.05)$. There were 3 cases of chest distress and palpitation discomfort in the treatment group, while 8 cases in control group, in which 2 cases had paroxysmal dyspnea. In Yang's study (Yang, 2016), all patients experienced leukopenia, gastrointestinal reactions and heart damage. The incidence of severe toxic and side effects was $25.00 \%$ in treatment group and $52.50 \%$ in control group, with a significant difference between two groups $(p<0.05)$. In Xie's study (Xie, 2014), there were 12 cases of bone marrow suppression and 2 cases of electrocardiogram changes in treatment group, while 19 cases of bone marrow suppression and 8 cases of electrocardiogram changes in control group. In Xiang's study (Xiang, 2019), there were 1 case of edema and 1 case of chest pain in treatment group, while 3 cases of edema and 3 cases of chest pain in control group. In Huang's study (Huang et al., 2008), 70.0\% of the patients in treatment group reached level 0 after treatment, while only $43.3 \%$ in control group, with statistically significant difference between two groups $(p<0.05)$. In Dai's study (Dai et al., 2007), the primary ADRs were bone marrow suppression and gastrointestinal reactions. Level IV side effects were not observed in two groups. In Chen's study (Chen X. C., 2016), there were 10 cases of bone marrow suppression and 1 case of electrocardiogram changes in treatment group, while 20 cases of bone marrow suppression and 6 cases of electrocardiogram changes in control group. In Mi's study (Mi, 2011), the incidence of degree III and degree IV myelosuppression was $60 \%$ in control group, while $16 \%$ in treatment group. The increasing trend of transaminase in control group was higher than that in treatment group, and the difference was not statistically significant. There were 7 cases of nausea and vomiting in control group and 19 cases in treatment group. In addition, fever symptoms also existed. In Ma's study (Ma et al., 2015), there were 2 cases of electrocardiogram changes in treatment group, while 6 cases in control group. In Wang's study (Wang W., 2015), there were 12 cases of decreased hemoglobin in the treatment group and 18 cases in control group. In Fu's study (Fu, 2014), there were 11 cases of decreased hemoglobin in the treatment group and 18 cases in control group. In Wang's study (Wang et al., 2006), no damage of liver and kidney function was found, and no phlebitis or rash occurred. Only three patients had low fever, which could be relieved by themselves after stoping the medicine.

CKI combined with CF was used as a treatment group intervention in seven RCTs: In Wang's study (Wang and Liu, 2007), there were 4 cases of bone marrow suppression, 0 cases of oral ulcer, and 3 cases of phlebitis in the treatment group. While 18 cases of bone marrow suppression, 5 cases of oral ulcer, and 4 cases of phlebitis in control group. Zhang's study (Zhang and Zhang, 2019) compared the scores of TCM symptoms before and after treatment. The treatment group was superior to control group in terms of fatigue, increased nocturia, lumbago pain, edema, and abdominal distention, and the differences between groups were statistically significant $(p<0.05)$. In Zhai's study (Zhai, 2014), there were 2 cases of oral mucositis in treatment group while 10 cases in control group. In Sun's study (Sun, 2009), only one patient had pain at the site of intravenous drip with flushed complexion, which was recovered after immediate discontinuation. In Ren's study (Ren et al., 2010), the decrease of WBC and abnormal transaminase in the treatment group was better than that in control group. In Wei's study (Wei, 2010), electrocardiogram changes after chemotherapy were observed in two groups, and level $\mathrm{I}$ and level II of toxic reactions in the 
A

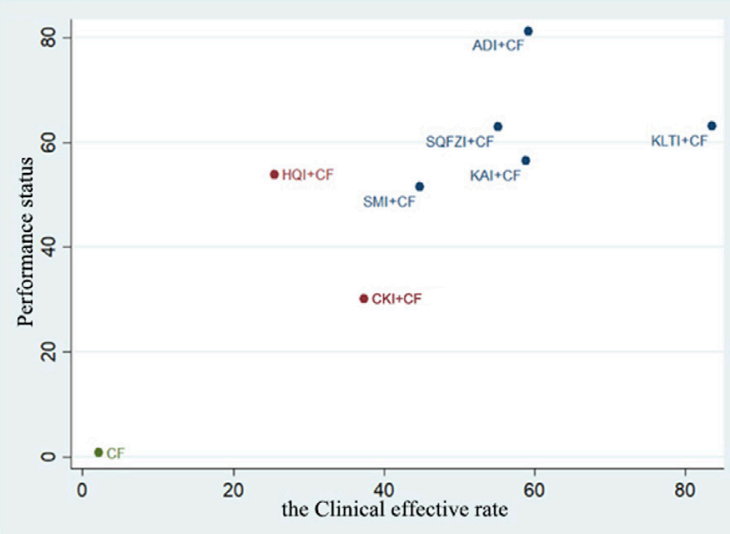

B

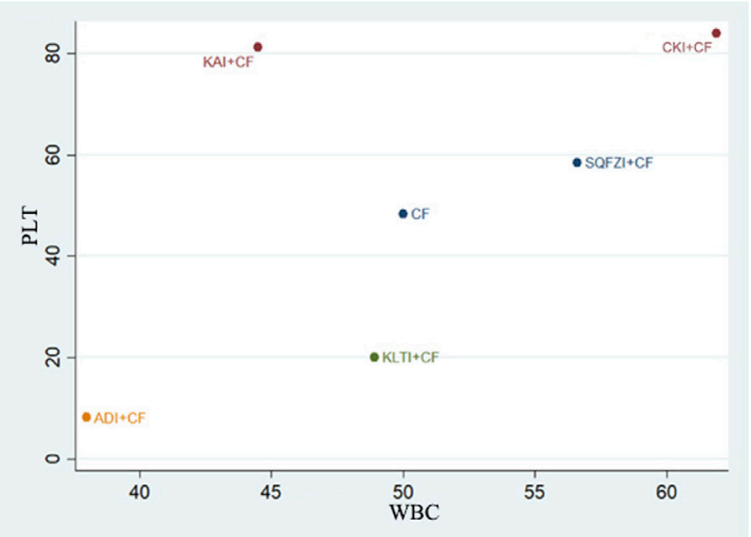

C

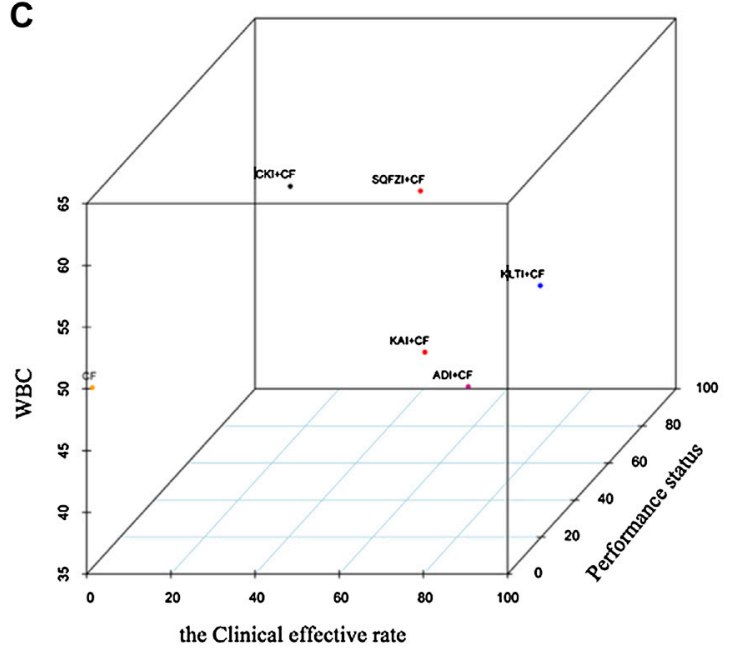

E

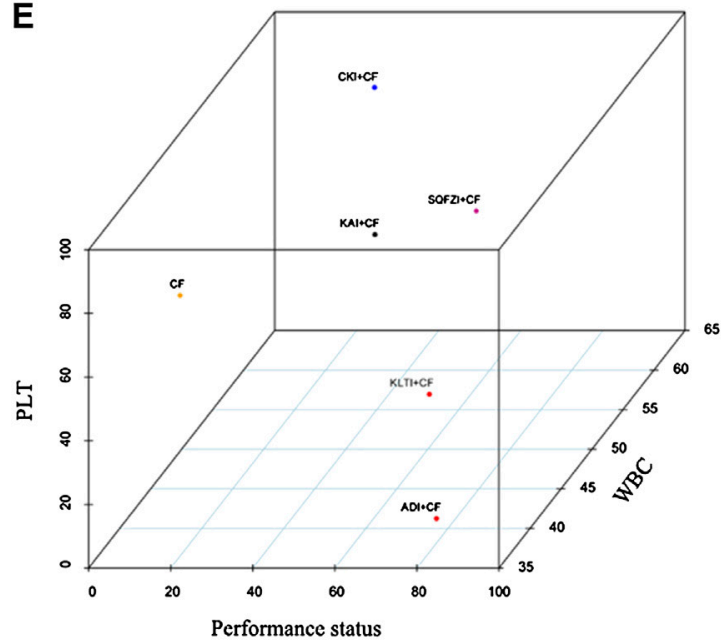

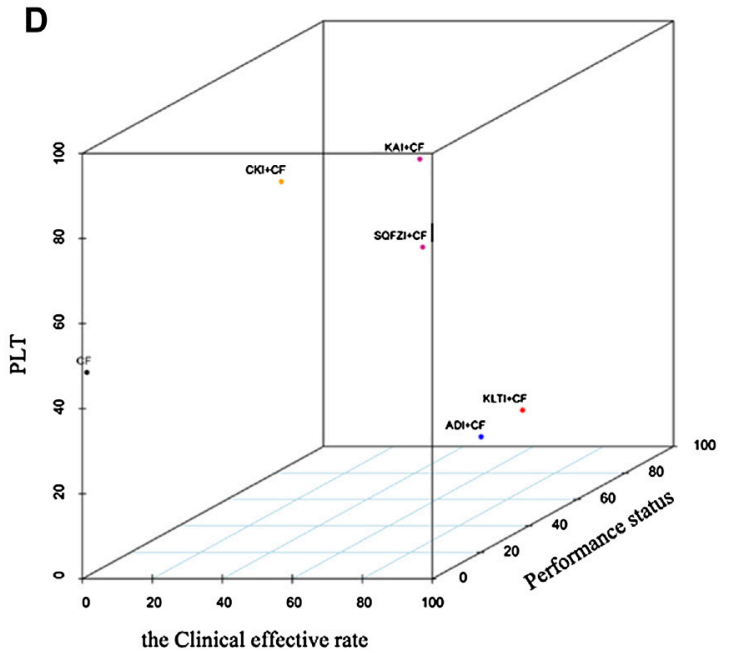

$\mathbf{F}$

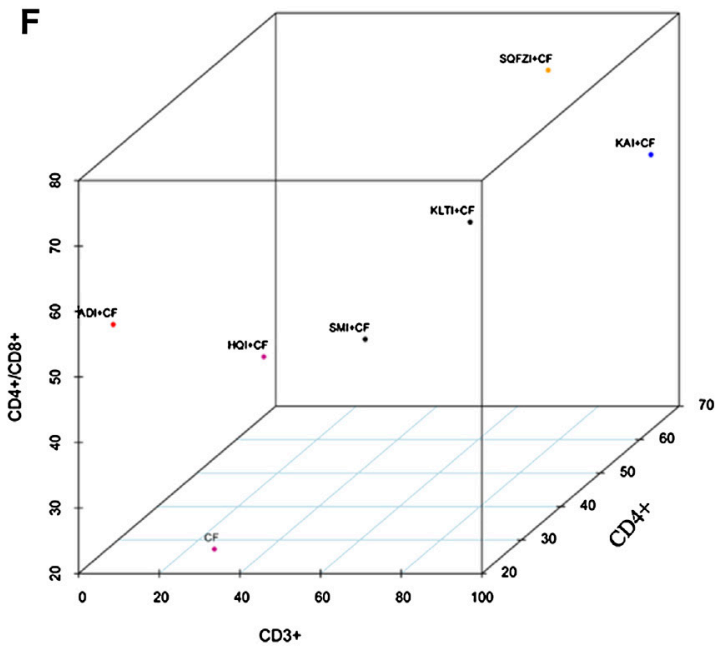

Figure 5 | Cluster analysis plot for outcomes. (A) Cluster analysis plot of the Clinical effective rate and Performance status; (B) Cluster analysis plot of the improvement of WBC and PLT; (C) Cluster analysis plot of the Clinical effective rate, improvement of WBC, and Performance status; (D) Cluster analysis plot of the Clinical effective rate, improvement of PLT, and Performance status; (E) Cluster analysis plot of Performance status, improvement of WBC, and PLT; (F) Cluster analysis plot of CD3 ${ }^{+}$, CD4+/CD8+, and CD4+. Note: Interventions with the same color belonged to the same cluster, and interventions located in the upper right corner indicate optimal therapy for two different outcomes; SQFZI, Shenqi Fuzheng injection; CKI, Compound Kushen injection; SMI, Shenmai injection; KAl, Kangai injection; ADI, Aidi injection; KLTI, Kanglaite injection; HQI, Huangqi injection; HCSI, Huachansu injection; SI, Shengmai injection; CF, Cyclophosphamide and 5-Fluorouracil. 
treatment group were 5 cases and 3 cases respectively, while level I, level II, and level III of toxic reactions in control group were 4 cases, 2 cases, and 2 cases respectively. The main manifestations of cardiotoxicity in two groups were sinus tachycardia and atrial arrhythmia.

HCSI combined with CF was used as a treatment group intervention in one RCT: In Song's study (Song, 2002), the cardiotoxicity in both treatment group and control group was sporadic. Liver and kidney function injuries were also rare, of which the incidence was lower in treatment group $(p<0.05)$. The incidence of venous stimulation was $61.5 \%$ in treatment group as well as $23.8 \%$ in control group. Patients can recover spontaneously within 3-5 days after drug withdrawal.

HQI combined with CF was used as a treatment group intervention in two RCTs: In Lu's study (Lu, 2010), there were seven patients had decreased PLT in treatment group and 14 patients in control group. There were five patients had abdominal pain in treatment group, while 10 patients in control group. In Huang's study (Huang et al., 2013), both treatment group and control group had 2 cases of level III and level IV platelet decline respectively.

KAI combined with CF was used as a treatment group intervention in 11 RCTs: In Shi's study (Shi et al., 2017), there were 2 cases of decreased PLT and 2 cases of fatigue in treatment group, while 9 cases and 8 cases in control group respectively. In Su's study (Su et al., 2016), there were 14 cases of granulocytopenia in treatment group and 30 cases in control group; 2 cases of fatigue in treatment group and 26 cases in control group; 4 cases of acute diarrhea in treatment group and 8 cases in control group. In Tan's study (Tan et al., 2018), there were 5 cases of thrombocytopenia in treatment group and 21 cases in control group. In Chen's study (Chen F. W., 2016), the incidence of ADRs in the treatment group was $24 \%$, including 1 case of nausea and vomiting, 1 case of abnormal liver function, 1 case of abnormal kidney function, 1 case of abnormal heart function, and 2 cases of WBC decline. The incidence of ADRs in the control group was $60 \%$, including 4 cases of nausea and vomiting, 5 cases of WBC reduction, 2 cases of abnormal liver function, 2 cases of abnormal kidney function, and 2 cases of abnormal heart function. The difference of ADRs of chemotherapy drugs in two groups were statistically significant $(p<0.05)$. In Wu's study (Wu and $\mathrm{Wu}, 2011$ ), there were 2 cases of abnormal cardiac function in treatment group and 3 cases in control group. In Zhu's study (Zhu and Sun, 2012), the major ADRs were leukopenia and digestive tract reactions, of which the specific number had not been specified.

\section{Cluster Analysis}

For primary outcome indicators, cluster analysis was used to evaluate the relative best treatment for $\mathrm{BC}$ in this study. Twodimensional clustering results indicated that ADI and CKI combined with $\mathrm{CF}$, at the position furthest from the zero point, were the best in improving the clinical effectiveness rate and performance status. CKI combined with CF was the preferred treatment to improve the decline of WBC and PLT. Three-dimensional clustering results indicated that SQFZI combined with CF was the preferred treatment in the respect of simultaneously improving the clinical effectiveness rate, performance status, and increasing WBC. KAI combined with $\mathrm{CF}$ was the best method to improve the clinical effectiveness rate, performance status, and increasing PLT. CKI and SQFZI combined with CF were both ideal treatments in terms of improving the performance status, increasing WBC, and increasing PLT. SQFZI and KAI combined with $\mathrm{CF}$ were both ideal treatments in terms of improving the decline of $\mathrm{T}$ cell subsets $\mathrm{CD} 3+, \mathrm{CD} 4+$, and CD4+/CD8+ (Figure 5).
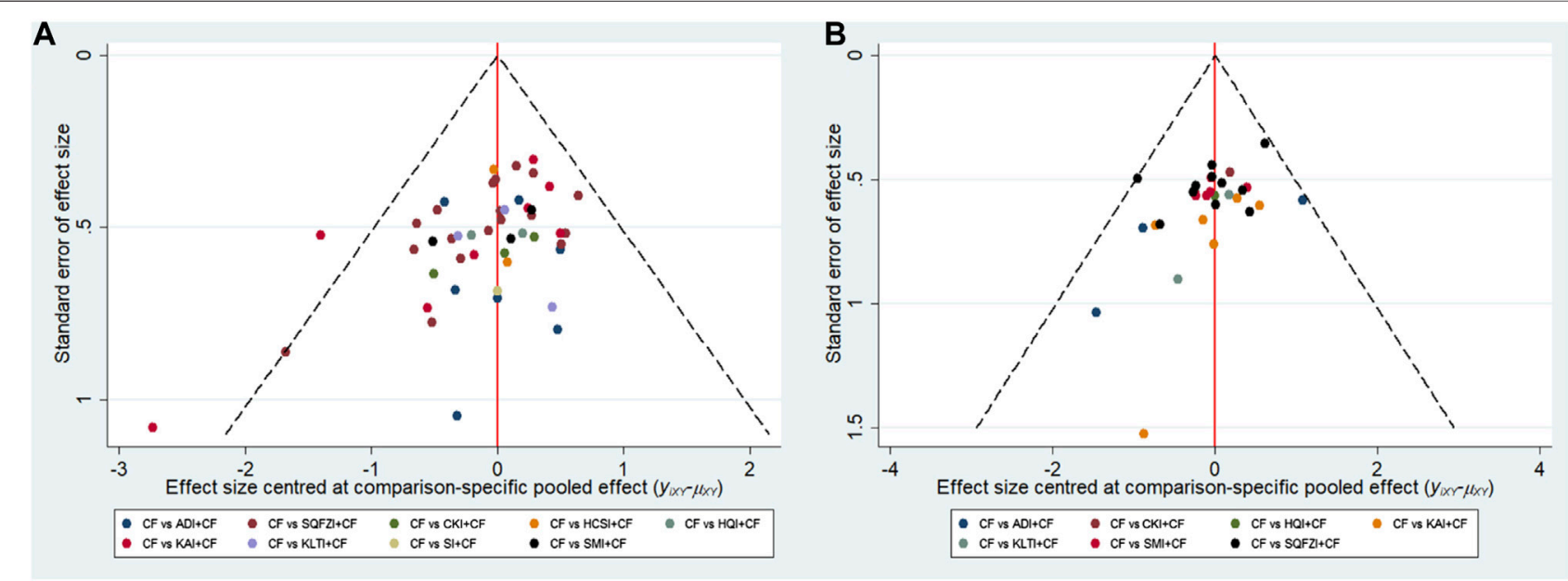

Figure 6 | Funnel plots. (A) Clinical effectiveness rate; (B) Performance status. Note: SQFZI, Shenqi Fuzheng injection; CKI, Compound Kushen injection; SMI, Shenmai injection; KAI, Kangai injection; ADI, Aidi injection; KLTI, Kanglaite injection; HQI, Huangqi injection; HCSI, Huachansu injection; SI, Shengmai injection; CF, Cyclophosphamide and 5-Fluorouracil. 


\section{Publication Bias}

Aiming at the main outcome indicators, this study drawn funnel polts for the clinical effectiveness rate and performance status. Funnel plots of the two outcomes were not completely symmetrical, which indicated that there was a certain publication bias in this study (Figure 6).

\section{DISCUSSION}

Based on the NMA method, this study comprehensively evaluated the efficacy and safety of nine CHIs combined with $\mathrm{CF}$ chemotherapy in the treatment of BC. The results of the study indicated that compared with the CF alone, ADI, SMI, SQFZI, KAI, KLTI, and SI combined with CF yielded significantly higher probability of improving the clinical effectiveness rate of $\mathrm{BC}$ patients. ADI, SMI, SQFZI, CKI, KAI, and KLTI plus CF were significantly associated with better performance status. The combination of $\mathrm{ADI}$ and $\mathrm{CF}$, KAI and CF could more effectively alleviate the reduction of PLT. CF combined with SQFZI could retard the decline of $\mathrm{T}$ cell subsets CD3+, CD4+, $\mathrm{CD} 8+$, and $\mathrm{CD} 4+/ \mathrm{CD} 8+$ in $\mathrm{BC}$ patients. With regard to ADRs, CF combined with ADI, SQFZI, and KAI seemed to be the optimal for significantly relieving gastrointestinal reactions. CF combined with SQFZI, CKI, and KAI could better moderate liver and kidney dysfunction. The combined use of CF and KAI was more effective in reducing hair loss symptoms. SMI, SQFZI, HQI, and $\mathrm{KAI}$ with $\mathrm{CF}$ can relieve leukopenia compared with using $\mathrm{CF}$ alone. ADI, SMI, SQFZI, CKI, HQI, and KAI could significantly alleviate the symptoms of nausea and vomiting. Furthermore, the results of cluster analysis suggested that compared with other CHIs, ADI, and CKI with CF for treating $\mathrm{BC}$ had its preponderance in improving the clinical effectiveness rate and performance status.

CHIs is a kind of sterile preparation for injection into the human body that is extracted and purified from TCM or prescription Chinese medicine, guided by the TCM theory and using modern technology (National Pharmacopoeia Commission, 2015). Compared with traditional decoction and other oral dosage forms, CHIs has the advantages of rapid onset and high bioavailability, aside from the characteristics of TCM syndrome differentiation treatment ( $\mathrm{Xu}$ et al., 2018). Some studies have pointed out that ADI can significantly reduce the level of blood CEA and CA199 in patients with gastrointestinal tumors, improve the level of T lymphocyte subgroup, enhance NK cell activity, and improve the performance status of patients (Zhang and Yang, 2009; Deng et al., 2011). The conclusion is consistent with the results of this study. Pharmacological researches have confirmed that SMI contains ginsenoside, Ophiopogon japonicus saponin, ginseng polysaccharide, Ophiopogon japonicus polysaccharide and other active ingredients, which can enhance the anti-tumor ability of tumor patients and have a protective effect on the human hematopoietic system. The mechanism of alleviating leukopenia and enhancing the immune function of patients is related to the role of total ginsenosides in stimulating the proliferation and differentiation of bone marrow hematopoietic stem cells (Feng and Liu, 1995). SQFZI is composed of astragalus and codonopsis with the effects of improving qi deficiency, as well as regenerating and nourishing blood. Astragalus contains various active ingredients such as astragalus polysaccharides, amino acids and glycosides, which can effectively enhance the body's specific immune function and promote the secretion of cytokines. Codonopsis has the function of anti-oxidation and relieving bone marrow toxicity (Wang et al., 2016). CKI has the efficacy of clearing away heat and detoxification, and has a certain inhibitory effect on the growth of tumor cells. The main component, matrine, can inhibit MMP-9 and MMP-2 activation in highly metastatic human breast cancer MDA-MB-231 cells, reduce the mRNA expression levels of MMP-9 and MMP-2, and finally inhibiting tumor cell invasion (Wang, 2018). Studies have shown that CKI can inhibit mouse sarcoma growth by inhibiting ERK, AKT kinase, and BAD phosphorylation (Dai et al., 2019). HCSI is made of toad skin through special processing. Research shows that it can not only significantly improve the phagocytic capacity of macrophages, but also enhance the patient's immunity, reduce bone marrow suppression, peripheral neurotoxicity, gastrointestinal reactions and other adverse effects (Wang and Lu, 2018). The main components of HQI are flavonoids, saponins, amino acids and other secondary metabolites, which have various pharmacological effects, such as enhancing myocardial contractility, increasing the content of superoxide dismutase in the body, scavenging oxygen free radicals, improving microcirculation, lowering blood sugar and blood fat, and regulating immune function (Duan et al., 2017). The main components of KAI are matrine, astragalus, and ginseng. Matrine can inhibit tumors and vascular endothelial cells, and can clear free radicals. Ginsenoside can promote the expression of bone marrow stromal cells, macrophages, and interleukin-6. Astragalus has the effect of regulating and protecting the immune system, renal function, and cardiovascular (Li P P, 2018). KLTI is an injection extracted from coix seed with anti-tumor activity. Modern pharmacological research shows that KLTI can down-regulate $\mathrm{Bcl}-2$ and up-regulate p53 gene expression to inhibit tumor growth and metastasis, adjust the expression of NF- $\kappa \mathrm{B}, \mathrm{IL}-2$ and other cytokines, promote $\mathrm{T}$ cell proliferation, and improve the body's immunity (Chen et al., 2019). SI is mainly composed of red ginseng, ophiopogon japonicus, and schisandra chinensis. Pharmacological researches have shown that it can inhibit the glycolysis energy supply pathway that tumor cells depend on, enhance the efficacy of drugs, reduce the side effects of chemotherapy, and increase immune cell attack (Hou et al., 2011).

In recent years, the incidence of $\mathrm{BC}$ has continued to rise. This is a heavy burden for some developing countries, in which screening and awareness programs are seriously lacking (Nassar et al., 2020). BC is usually called "stone breast" in TCM theory, and in which the pathogenesis of $\mathrm{BC}$ is considered to be related to the intermingling of factors such as emotional disorder, Liver-kidney Yin Deficiency, Qi stagnation and blood stasis, and irregular diet (Xie and Long, 2019; Xu and Luo, 2019). CHIs have the functions of improving the internal environment, adjusting the congenital functions, and enhancing the anti-cancer ability of human body. The application of CHIs in clinical adjuvant treatment of cancer is increasingly widespread. So far, only Tian Jinhui's team has reported a NMA regarding CHIs combined with CF against BC. The study included 26 RCTs, 
involving 1884 patients and a total of six kinds of CHIs. NMA results showed that CKI and KLTI combined with CF were superior to other programs in improving the clinical effectiveness rate and performance status of patients. SQFZI and ADI combined with CF chemotherapy were more effective in reducing the incidence of nausea and vomiting (grade III to IV) and decreased WBC (grade III to IV). In our study, we added three kinds of CHIs (HQI, SMI and SI) and 58 RCTs, focusing on two new outcomes of T-lymphocyte subsets and peripheral hemogram, meta-analysis results indicated that the evidence is more credible.

At the same time, this study also has several limitations. First of all, the 84 studies included were only discussed the Chinese patients, which caused publication bias to a certain extent. Secondly, the patient's TNM classification and course of disease are different, which may lead to an imbalance between studies. Thirdly, the number of RCTs involved in each CHI is different. For injections with a small sample size, the clinical efficacy of which may be exaggerated, which may bias the results. Finally, this study performed a risk of bias evaluation of the included studies, and the results showed that the quality of them was not very high. A part of the included studies did not specify the specific randomization method, and almost all the studies did not mention the implementation of blinding and allocation concealment. This further affected the credibility of the results of each outcome, and directly led to the low quality of the evidence in this study. Many previous methodological studies have shown that if the design or implementation of clinical trials is flawed, there would be a great risk of causing misleading results, and may even exaggerate the clinical efficacy of interventions. The implementation of blinding and allocation concealment will greatly reduce the bias of the results caused by the subjective ideas of trial participants. Therefore, although the original researches included in this study are RCTs, which are often considered to be the highest quality of evidence, there is limited confidence in the estimated value of the outcome in this study, and its true value may deviate from the estimated value. Given the above limitations, it is recommended that clinical RCTs should be registered in advance according to the Consort

\section{REFERENCES}

Bian, Z. Y., Tang, Q. Z., Li, F. F., and Wei, W. (2011). Systematic Reviews of the therapeutic effect of Huangqi on dilated cardiomyopathy. J. Medical Res. 40 (11), 70-72. doi:10.3969/j.issn.1673-548X.2011.11.022

$\mathrm{Bu}, \mathrm{X}$. Q., and Zhang, J. P. (2018). Effect of Shenmai injection on preventing myocardial injury in patients undergoing chemotherapy after breast cancer surgery. Chin. J. Integr. Med. Cardio/Cerebrovasc. Dis. 16 (23), 3506-3508. doi:10.12102/j.issn.1672-1349.2018.23.032

Cao, Y. L., Li, Z. H., Lei, Q. M., Chen, G., Gong, Y., Lei, S. G., et al. (2009). Effect of Kangai injection used with breast cancer chemotherapy and postoperation. Cancer Res. Prev. Treat. 36 (3), 241-243. doi:10.3971/j.issn. 1000-8578.2009.03.021

Chen, F., and Lin, H. (2007). Clinical observation of Shenqi Fuzheng injection in assist chemotherapy of breast cancer. Strait Pharm. J. 19 (12), 75-76. doi:10. 3969/j.issn.1006-3765.2007.12.039

Chen, F. W. (2016). Clinical study of Kang'ai injection combining with CEF in sequence and docetaxel for chemotherapy in the treatment of breast standard to ensure the standardization and transparency of the trial process. At the same time, random group hiding and blind methods should be implemented as much as possible to report the test results truthfully. The results of large-sample, multi-center, randomized double-blind controlled trials have higher credibility. Finally, when conducting clinical research on the treatment of BC with CHIs, patients can be diagnosed and treated according to TCM syndromes while adopting western medicine diagnostic criteria to reflect the characteristics of TCM.

\section{CONCLUSION}

In conclusion, compared with using $\mathrm{CF}$ alone, the combination of $\mathrm{CHIs}$ and $\mathrm{CF}$ was associated with improved treatment performance and safety, and could be beneficial for patients with BC. ADI and KLTI were noteworthy in improving the clinical effectiveness rate and performance status. SQFZI and KAI have better efficacy in reducing ADRs caused by chemotherapy. However, more highquality clinical RCTs, especially which correctly use blinding and allocation concealment, are required to support the conclusions.

\section{AUTHOR CONTRIBUTIONS}

JW and SL done conception and design of the network metaanalysis; SL, HW and MN performed the network meta-analysis; SL, XZ, and JW assessed the quality of the network meta-analysis; SL, HW, and MW analyzed study data; SL, HW, XH, WY, RJ, and YG wrote the paper. All authors read and approved the final version of the manuscript.

\section{FUNDING}

This work was supported by the National Nature Science Foundation of China (Grant Nos. 81473547, 81673829) and Young Scientists Training Program of Beijing University of Chinese Medicine.

cancer. China Mod. Med. 23 (22), 106-108. doi:CNKI:SUN:ZGUD.0.201622-037

Chen, L., Xiao, D., Liang, M., Zhai, C., Zhang, Y. L., Zheng, M. H., et al. (2018). Effect of Shenmai injection on serum PCT IL-6 TNF-alpha and myocardial function in patients with breast cancer after operation. Hebei Med. 24 (4), 623-627. doi:10.3969/j.issn.1006-6233.2018.04.025

Chen, Q. S., Liu, J. B., Huang, Z. F., and Huang, C. J. (2010). Shenmai injection combined with chemotherapy for advanced breast cancer. West China Med J. 25 (3), 597-598. doi:CNKI:SUN:HXYX.0.2010-03-078

Chen, S. S., Liu, F. F., Liu, Z. S., Zhang, X. F., and Wei, L. (2019). Effects of kanglaite on tumor markers and quality of life of patients with advanced breast cancer. Anti-Tumor Pharm. 9 (2), 300-303. doi:10.3969/j.issn.20951264.2019.02.26

Chen, W. J., and Chen, C. (2013). Radix astragali in the treatment of dilated cardiomyopathy: a meta-analysis. Guide China Med. 11 (17), 468-471. doi:10. 3969/j.issn.1671-8194.2013.17.357

Chen, W. M. (2016). Clinical observation of Aidi injection combined with CEF chemotherapy in the treatment of postoperative breast cancer. Chin. J. Mod. Drug Appl. 10 (15), 185-187. doi:10.14164/j.cnki.cn11-5581/r.2016.15.134 
Chen, W. Q., Zheng, R. S., Baade, P. D., Zhang, S. W., Zeng, H. M., Bray, F., et al. (2016). Cancer statistics in China. Ca-Cancer J. Clin. 66 (2), 115-132. doi:10. $3322 /$ caac. 21338

Chen, X. C. (2016). Clinical effect of Shenqi Fuzheng injection combined with chemotherapy for breast cancer. World Latest Med. Inform. Digest. 16 (91), 144-150. doi:10.3969/j.issn.1671-3141.2016.91.113

Chen, Y. F. (2005). Application of Shengmai injection in breast cancer chemotherapy. J. Snake. (4), 267-268. doi:10.3969/j.issn.1001-5639.2005.04.019

Dai, Y. N., Han, J. J., Li, J. M., Chen, X. J., Feng, X. X., and Wang, R. (2019). Clinical observation on compound Sophora flavescens injection combined with neoadjuvant chemotherapy on primary breast cancer. Liaoning J. Tradit. Chin. Med. 46 (10), 2121-2123. doi:10.13192/j.issn.1000-1719.2019.10.033

Dai, Z. J., Wang, X. J., Kang, H. F., Guan, H. T., Liu, X. X., Song, L. Q., et al. (2007). Clinical observation of efficacy and adverse reactions on Shenqifuzheng injection used in patients with breast cancer receiving neoadjuvant chemotherapy. Adv. Drug Reactions J. (1), 10-14. doi:10.3969/j.issn.10085734.2007.01.003

Dang, X. G., and Wang, L. (2010). Evaluation of efficacy on breast cancer treated by Aidi injection plus CTF program of neoadjuvant chemotherapy and the impacts on serum sFas. World J. Integr. Tradit. West. Med. 5 (1), 54-56. doi:10.3969/j. issn.1673-6613.2010.01.021

Deng, A. P., Tang, Q., Fu, T. X., and Wang, Y. (2011). The effects of AD injection on tumor marker in alimentary tract malignant tumors. Chin. J. Clin. Oncol. Rehabil. 18 (2), 123-124. doi:CNKI:SUN:ZGZK.0.2011-02-008

Diao, H. Y., Yang, G. H., and Han, Y. G. (2018). Effect of Shenqi fuzheng injection on quality of life and immune function of patients after breast cancer chemotherapy. Henan Med. Res. 27 (20), 3686-3689. doi:10.3969/j.issn. 1004-437X.2018.20.013

Dong, F. F., and Ren, Q. Y. (2018). Research on TCM syndrome and treatment of breast cancer. Chin. J. Ethnomed. Ethnopharmacy. 93 (1), 22-23.

Duan, X. J., Wu, J. R., Wang, K. H., Liu, S., Zhang, D., Zhang, X. M., et al. (2017). Meta-analysis of efficacy of Huangqi injection in the treatment of cerebral infarction. Chin. J. Pharmacoepidemiol. 26 (9), 607-612. doi:CNKI:SUN: YWLX.0.2017-09-008

Fan, L., Strasser-Weippl, K., Li, J. J., St Louis, J., Finkelstein, D. M., Yu, K. D., et al. (2014). Breast cancer in China. Lancet Oncol. 15 (7), 279-289. doi:10.1016/ S1470-2045(13)70567-9

Feng, P. F., and Liu, L. M. (1995). Effect of Shenmai injection on sIL-2R, LAK and NK cell activity in patients with advanced cancer. Chin. J. Integr. Tradit. West. Med. 15 (2), 87-89. doi:10.7661/CJIM.1995.2.87

Fu, B. H., Li, Q. H., Han, T., Guo, F., Zhang, J., Liu, Z. Z., et al. (2015). Clinical observation of kanglaite injection combined chemotherapy in treatment of breast cancer. Clin. Misdiagn. Misther. 28 (12), 57-60. doi:10.3969/j.issn.10023429.2015.12.023

Fu, Y. J. (2014). Observation of the curative effect of Chinese and Western medicine combined on breast cancer. China Pract. Med. 9 (17), 138-139. doi:CNKI:SUN: ZSSA.0.2014-17-105

Gan, L. L., Yu, J. P., Zhang, H. Q., and Shi, L. G. (2009). Role of Kanglaite injection in the neoadjuvant chemotherapy of breast cancer. TUMOR. 29 (3), 283-285. doi:10.3781/j.issn.1000-7431.2009.03.020

Gao, L., Li, Z. X., Yang, J., Wu, S. S., Wang, W. W., Yang, Z. R., et al. (2018). Impact of glucagon-like peptide-1 receptor agonists on headache and dizziness among type 2 diabetes: a network meta-analysis. Chin. J. Pharmacoepidemiol. 27 (1), 7-13. doi:CNKI:SUN:YWLX.0.2018-01-002

Gao, Y., and Wang, X. X. (2013). Viewing the transformation of differentiation and treatment of breast cancer from the theory of "sui qi suo de". J. Tradit. Chin. Med. 54 (16), 1434-1436. doi:CNKI:SUN:ZZYZ.0.2013-16-036

Ge, L., Pan, B., Pan, J. X., Li, Y. N., Wu, Y. T., Guo, S. J., et al. (2017). An introduction of AMSTAR-2 : a quality assessment instrument of systematic Reviews including randomized or non-randomized controlled trials or both. Chin. J. Drug Eval. 34 (5), 334-338. doi:CNKI:SUN:YAPJ.0.201705-004

Gu, X. D., Zhao, H., and Xie, X. H. (2015). Observation of curative effect of compound Kushen injection combined with neoadjuvant chemotherapy on patients with locally advanced breast cancer. Zhejiang J. Tradit. Chin. Med. 50 (1), 74. doi:CNKI:SUN:ZJZZ.0.2015-01-060

Guo, Z. H., and Hu, Q. L. (2018). Effects of Shenqi Fuzheng injection combined with chemotherapy on peripheral blood, im-mune function and tumor markers in patients with breast cancer. J. Hainan Med. Univ. 24 (17), 1599-1602. doi:10. 13210/j.cnki.jhmu.20180725.004

Han, Y. H., Jiang, X. Q., Yang, H., Wu, Z. Y., and Jiang, M. (2014). Clinical observation of Aidi InJection combined with CEF regimen chemotherapy in treatment of postoperative breast cancer. Hubei J. Tradit. Chin. Med. 36 (5), 7-8. doi:CNKI:SUN:HBZZ.0.2014-05-005

He, Y. C. (2015). Study on the effect of adjuvant therapy of breast cancer patients undergoing chemotherapy with Shenqi Fuzheng injection. Contemp. Med. Forum. 13 (22), 267-268. doi:CNKI:SUN:QYWA.0.2015-22-228

Hou, X. F., Li, Z. Y., Fan, H., and Wang, H. (2011). Clinical observation on attenuated efficiency of Shengmai Injection in breast cancer chemotherapy. China Med. Herald. 8 (23), 90-92. doi:10.3969/j.issn.1673-7210.2011.23.043

Huang, C. J., Liu, J. B., Cai, K., and Liao, T. H. (2009). Clinical observation on treatment of 30 cases of advanced breast cancer with Shenmai injection and chemotherapy. Chin. J. Tradit. Med. Sci. Technol. 16 (2), 144-145. doi:10.3969/j. issn.1005-7072.2009.02.031

Huang, C. J., Liu, J. B., and Liao, T. H. (2013). Effect of Astragalus injection combined chemotherapy on life quality and immune function in advanced breast cancer. Inner Mongol J. Tradit. Chin. Med. 32 (32), 66-67. doi:10.3969/j. issn.1006-0979.2013.32.076

Huang, Z. F., Wei, J. S., Li, H. Z., Tan, Z. Q., Zhang, Z. J., and Chen, C. (2008). Effect of Shenqi fuzheng injection combined with chemotherapy on thirty patients with advanced breast cancer. Chin. J. Integr. Tradit. West. Med. 28 (2), 152-154. doi:10.3321/j.issn:1003-5370.2008.02.014

Hutton, B., Salanti, G., Caldwell, D. M., Chaimani, A., Schmid, C. H., Cameron, C., et al. (2015). The PRISMA extension statement for reporting of systematic Reviews incorporating network meta-analyses of health care interventions: checklist and explanations. Ann. Intern. Med. 162 (11), 777-784. doi:10.7326/ M14-2385

Jia, C. F., Duan, M., and Duan, X. (2016). Clinical effects of Shen Qi Fu Zheng injection and chemotherapy on hematopoietic and im-mune function in patients with breast cancer. J. Hainan Med. Univ. 22 (16), 1866-1869. doi:10.13210/j.cnki.jhmu.20160602.002

Jia, Q. L., Hu, Y. H., Song, Q. Q., Du, B., Li, K., and Li, S. S. (2015). A systematic review on the efficacy and safety of Shenfu injection in the treatment of dilated cardiomyopathy. Chin. J. Evidence Based Cardiovascular Med. 7 (5), 609-613. doi:10.3969/j.issn.1674-4055.2015.05.08

Jiang, X. M., Jia, X. J., Yi, Y., Huang, X. R., Lei, H., and Luo, X. (2017). Study on the effect of Kanglaite injection combined with chemotherapy on T cell subsets and tumor markers in patients with breast cancer. Chin. J. Health Lab. Technol. 27 (17), 2527-2529. doi:CNKI:SUN:ZWJZ.0.2017-17-028

Jinatongthai, P., Kongwatcharapong, J., Foo, C. Y., Phrommintikul, A., Nathisuwan, S., Thakkinstian, A., et al. (2017). Comparative efficacy and safety of reperfusion therapy with fibrinolytic agents in patients with ST-segment elevation myocardial infarction: a systematic review and network meta-analysis. Lancet. 390 (10096), 747-759. doi:10.1016/S0140-6736(17)31441-1

Lai, Y. F., Lu, J. J., Chen, X. P., Hu, H., and Wang, Y. T. (2012). Analysis of antitumor Chinese medicine injection based on market conditions. World Sci. Tech.-Modern. Trad. Chin. Med. (5), 1958-1962. doi:10.3969/j.issn.1674-3849. 2012.05.003

Lao, G. Q., Chen, F., Shi, Z. Y., Luo, J. H., and Liang, D. R. (2011). 30 cases of breast cancer treated by Shenmai injection combined with chemotherapy. Chin. Med. Mod. Distance Edu. China. 9 (16), 55-56. doi:10.3969/j.issn.1672-2779.2011. 16.037

Leucht, S., Cipriani, A., Spineli, L., Mavridis, D., Orey, D., Richter, F., et al. (2013). Comparative efficacy and tolerability of 15 antipsychotic drugs in schizophrenia: a multiple-treatments Meta-analysis. Lancet. 382 (9), 951-962. doi:10.1016/S0140-6736(13)60733-3

Li, J., Tang, Q. Z., Zhang, W. B., Xiang, S. Z., and Hu, Z. F. (2014). Meta analysis of the therapeutic effect of Shengmai injection on dilated cardiomyopathy. Chin. J. Evid. Based Cardiovascular Med. 6 (4), 382-385. doi:10.3969/j.1674-4055. 2014.04.03

Li, P. P. (2018). Observation of clinical effect of Kangai injection combined with NP regimen in the treatment of breast cancer. Henan Med. Res. 27 (20), 3794-3795. doi:10.3969/j.issn.1004-437X.2018.20.082

Li, S. (2018). Analysis of clinical curative effect of shenqifuzheng injection combined with new adjuvant chemotherapy in treatment of breast cancer. Syst. Med. 3 (22), 26-28. doi:10.19368/j.cnki.2096-1782.2018.22.026 
Li, X. L., Wang, X. Q., and Qu, H. O. (2015). Effect of Shenqifuzheng injection combined with chemotherapy on breast cancer. Chin. J. Clin. Pharmacol. 31 (4), 267-269. doi:10.13699/j.cnki.1001-6821.2015.04.009

Li, X. Q., and Gong, S. B. (2006). Analysis of curative effect of Aidi injection combined with CEF chemotherapy on breast cancer. Pharmacol. Clin. Chin. Materia Med. 22 (Z1), 176-177. doi:10.3969/j.issn.1001-859X.2006.03.092

$\mathrm{Li}, \mathrm{Y}$. X. (2012). Clinical observation on 60 cases of advanced breast cancer treated by compound kushen injection combined with chemotherapy. Guide China Med. 10 (36), 302-303. doi:10.3969/j.issn.1671-8194.2012.36.227

Li, Z. Y., Huang, Q. H., Wang, Y. H., and Ruan, X. F. (2006). Observation of curative effect of Kangai injection combined with chemotherapy on breast cancer. Chin. J. Clin. Oncol. Rehabil. 13 (6), 544-545. doi:10.3969/j.issn.10058664.2006.06.026

Liang, F., Zhang, Q. S., Zhang, L. J., Xing, G. C., and Li, J. M. (2014). Meta-analysis of Shenqi Fuzheng injection combined with chemotherapy for breast cancer. China Pract. Med. 9 (3), 175-176. doi:CNKI:SUN:ZSSA.0.2014-03-137

Liu, J. B., Huang, C. J., Cai, K., and Liao, T. H. (2009). Effect of Shenmai injection combined with chemotherapy on quality of life and immune function of patients with advanced breast cancer. Hebei J. Tradit. Chin. Med. 31 (9), 1346-1347. doi:10.3969/j.issn.1002-2619.2009.09.043

Liu, J. G. (2012). Systematic review on Shenmai injection treatment of dilated cardiomyopathy. Chin. Tradit. Patent Med. 34 (8), 1456-1461. doi:10.3969/j. issn.1001-1528.2012.08.010

Liu, Q., Zhang, X. F., Cheng, X. F., Wang, W., and Zhao, H. D. (2014). Clinical observation of cardiac toxicity induced by FAC regimen in female breast cancer treated by Shenmai injection. China J. Chin. Med. 29 (8), 1090-1091. doi:CNKI: SUN:HNZK.0.2014-08-002

Liu, X. Y., and Song, X. L. (2017). Effect of Shenqi Fuzheng injection on the immune function and quality of life of patients with breast cancer. Shanghai Med. Pharm. J. 38 (19), 29-31. doi:10.3969/j.issn.1006-1533. 2017.19.011

Liu, Y. (2017). Analysis of clinical effects of different drugs on breast cancer. Clin. Res. Pract. 2 (3), 16-17. doi:10.19347/j.cnki.2096-1413.201703008

Liu, Z. Z., Liu, H., Li, L. F., and Cui, S. D. (2005). Clinical observation of Aidi injection combined with chemotherapy in the treatment of locally advanced breast cancer. Shandong Med. J. 45 (19), 62. doi:10.3969/j.issn.1002-266X.2005.19.046

Lu, M. Y., Li, H. S., and Yang, B. (2010). The effects of shenqifuzheng injection on the immune function of patients with breast cancer treated with CAF regimen chemotherapy. J. Basic Clin. Oncol. 23 (3), 236-238. doi:10.3969/j.issn.16735412.2010.03.019

Lu, X. A. (2010). Effect of Huangqi injection on quality of life of breast cancer patients. Chin. Med. Mod. Dis. Educ. China. 8 (11), 36-37. doi:10.3969/j.issn. 1672-2779.2010.11.026

Ma, F. L., Li, S. B., Yin, Z., Li, J. M., and Liu, C. J. (2015). Clinical observation on 36 cases of breast cancer treated by Shenqi fuzheng injection combined with chemotherapy. Pharmacol. Clin. Chin. Mater. Med. 31 (2), 109-110. doi:CNKI: SUN:ZYYL.0.2015-02-045

Mi, C. (2011). Clinical value of Shenqi Fuzheng injection in preventing and treating side effects of breast cancer chemotherapy. Shijiazhuang, China: Hebei Medical University.

Nassar, F. J., Chamandi, G., Tfaily, M. A., Zgheib, N. K., and Nasr, R. (2020). Peripheral blood-based biopsy for breast cancer risk prediction and early detection. Front. Med. 7, 28. doi:10.3389/fmed.2020.00028

National Pharmacopoeia Commission (2015). Pharmacopoeia of the People's Republic of China. Beijing, China: China Medical Science and Technology Press

Pan, L. M. (2011). Analysis of 160 cases of advanced breast cancer combining cinobufacin and chemotherapy. China Foreign Med. Treatment. 30 (1), 59-61. doi:10.3969/j.issn.1674-0742.2011.01.038

Pan, Q., He, D. C., and Ding, X. J. (2008). Clinical observation on treatment of breast cancer with kang'ai injection combined with chemotherapy. Hubei J. Tradit. Chin. Med. 10, 14-15. doi:10.3969/j.issn.1000-0704.2008.10.006

Pan, Q., Yu, H., and You, J. L. (2016). Effect of Kanglaite combined with chemotherapy on myelosuppression,immune function and tumor markers levels in patients with breast cancer. J. Hainan Med. Univ. 22 (17), 1992-1995. doi:10.13210/j.cnki.jhmu.20160503.005

Qi, Q. G., Yue, L., Wang, Y., Li, H., Gao, Y., Xu, Y. Y., et al. (2013). Impact of Shenqifuzheng combination chemotherapy on the expression levels of TNF- $\alpha$, CCL18 and IL-6 in the serum of breast cancer patients. Chin. J. New Drugs. 22 (10), 1196-1201. doi:CNKI:SUN:ZXYZ.0.2013-10-019
Qiu, D. M. (2016). Kang Yi injection of chemotherapy for breast cancer patients with postoperative observation. J. Changchun Univ. Tradit. Chin. Med. 32 (3), 526-527. doi:10.13463/j.cnki.cczyy.2016.03.031

Ren, J. H., Wang, H., Liu, L. M., Li, X. H., Zhang, F. M., and Zhang, X. Q. (2010). Clinical observation on matrine injection combined with "CAF" chemotherapy for one hundred and twenty-three patients with early breast carcinoma underwent modified radical mastectomy. Eval. Anal. Drug Use Hosp. China. 10 (8), 736-739. doi:CNKI:SUN:YYPF.0.2010-08-029

Ren, Z. G., and Zhang, F. (2005). High-dose Aidi injection and chemotherapy in treating 60 cases of mammary cancer. J. Linyi Med. College. 27 (5), 341-343. doi:10.3969/j.issn.1674-0947.2005.05.007

Shi, H. Y., Tian, Y. Z., Zhang, L. Y., and Shao, L. L. (2017). Observation on the efficacy of Kangai injection in preventing and treating the side effects of postoperative chemotherapy of breast cancer. Zhejiang J. Tradit. Chin. Med. 52 (6), 464. doi:10.3969/j.issn.0411-8421.2017.06.057

Siegel, R. L., Miller, K. D., and Jemal, A. (2019). Cancer statistics. 2019. Canc. J. Clinic. 69 (1), 7-34. doi:10.3322/caac.21551

Song, W. G. (2002). Clinical observation of Huachansu combined with chemotherapy in the treatment of advanced breast cancer. National Conference on Breast Diseases with Integrated Chinese and Western Medicine, Chinese society of integrated traditional chinese and western medicine, $158-160$

Song, W. G., Wang, Y. F., Yang, L. X., Zhang, Z., Li, G. Z., and Wang, R. L. (2006). Clinical observation of Aidi combined with chemotherapy in the treatment of recurrent and metastatic breast cancer. Chin. J. Canc. Prevent. Treat. 13 (16), 1275. doi:10.3969/j.issn.1673-5269.2006.16.022

Song, Z. J. (2004). Clinical observation on the effect of Shenqi fuzheng injection on increasing the effect and toxicity of postoperative chemotherapy for breast cancer. J. Chin. Physician. 32 (9), 62-63. doi:10.3969/j.issn.1008-1089.2004.09.049

Su, Z. X., Deng, Z. P., Sun, H. F., and Zhao, Z. (2016). The efficacy and safety analysis of Kang'ai injection in the treatment of breast cancer. J. Bengbu Med. College. 41 (10), 1288-1289. doi:10.13898/j.cnki.issn.1000-2200.2016. 10.009

Sun, S. H., and Zheng, X. B. (2005). Application of Shenqi fuzheng injection in postoperative chemotherapy for breast cancer. Chin. J. Integr. Tradit. West. Med. 25 (6), 544-545. doi:10.3321/j.issn:1003-5370.2005.06.018

Sun, X. M. (2009). Effects of Matrine on immune functions in patients with breast carcinoma undergoing chemotherapy after surgery operation. J. Tongji Univ. 30 (1), 117-120. doi:CNKI:SUN:TJIY.0.2009-01-033

Tan, X. Q., Chen, Q. F., Luo, X. B., and Hu, X. H. (2018). The efficacy and adverse reactions of Kangai injection combined with CTF regimen in neoadjuvant chemotherapy for breast cancer. China Mod. Doctor. 56 (13), 74-77. doi:CNKI: SUN:ZDYS.0.2018-13-022

Wang, C. T., and Li, J. W. (2018). Advances in research on prevention and treatment of breast cancer with traditional Chinese medicine. J. China Prescription Drug. 16 (2), 18-20. doi:10.3969/j.issn.1671-945X.2018.02.013

Wang, C. Y., Song, C. Y., and Shen, F. M. (2012). Study on the protective effect of Shenmai injection on adriamycin cardiotoxicity. Clin. Focus. 27 (13), 1169-1170. doi:10.3969/j.issn.1002-1108.2011.06.35

Wang, D. J. (2013). Clinical observation of Chinese and western drugs for the treatment of 76 cases of breast cancer. Med. Recapitulate. 19 (14), 2676-2678. doi:10.3969/j.issn.1006-2084.2013.14.066

Wang, F. (2015). The comparative analysis of different medical approaches in treatment of breast cancer. China Health Std. Manag. 6 (3), 238-239. doi:10. 3969/j.issn.1674-9316.2015.03.187

Wang, L., and Liu, J. (2007). Clinical observation of CTF scheme combined with Yanshu injection in neoadjuvant chemotherapy for breast cancer. China Med. Herald. (24), 55-97. doi:10.3969/j.issn.1673-7210.2007.24.037

Wang, L. R., Wang, H. C., and Cao, Y. (2016). Observation on therapeutic effect of Shenqi fuzheng injection on 60 cases of breast cancer. Chin. J. Cancer Prev. Treat. 23 (2), 113-114. doi:CNKI:SUN:QLZL.0.2016-S2-051

Wang, Q. Y., and Lu, Z. Y. (2018). The clinical efficacy of cinobufacini injection combined chemotherapy in the treatment of advanced breast cancer. China Foreign Med. Treat. 37 (6), 125-127. doi:10.16662/j.cnki. 1674-0742.2018.06.125

Wang, S. M., Guo, Y. W., and Huang, G. S. (2006). Application of Shenqi fuzheng injection in adjuvant chemotherapy after breast cancer operation. Med. Industry Inform. 3 (17), 124. doi:10.3969/j.issn.1673-7210.2006.17.094 
Wang, W. (2015). Clinical study of Shenqi fuzheng injection combined with new assistant chemotherapy on breast cancer. China J. Chin. Med. 30 (4), 466-467. doi:10.16368/j.issn.1674-8999.2015.04.159

Wang, Y., and Wang, K. Y. (2011). Clinical effect of Kangai injection on breast cancer. Chin. J. Mod. Drug Appl. 5 (21), 59-60. doi:10.3969/j.issn.1673-9523. 2011.21.051

Wang, Y. X. (2018). Clinical observation of compound Kushen injection combined with chemotherapy in the treatment of triple negative breast cancer. World Latest Med. Inform. Dig. 18 (19), 3-4. doi:CNKI:SUN:WMIA.0.2018-19-002

Wei, Y. H. (2010). 24 cases of compound Kushen injection combined with adjuvant chemotherapy after breast cancer operation. Chin. Med. Mod. Dis. Educ. China. 8 (8), 100-101. doi:10.3969/j.issn.1672-2779.2010.08.077

Wu, F. (2010). Observation of the clinical observation of Kangai injection combined with accessory chemotherapy after breast cancer operation. World J. Integr. Tradit. West. Med. 5 (6), 522-524. doi:10.3969/j.issn.1673-6613.2010.06.023

Wu, J. R., Hu, X., Wang, K. H., Duan, X. J., Zhang, D., and Ni, M. W. (2017). Metaanalysis on Shenmai injection in treatment of dilated cardiomyopathy. Eval. Anal. Drug-Use Hosp. China. 17 (11), 1448-1452. doi:10.14009/j.issn.16722124.2017.11.003

$\mathrm{Wu}, \mathrm{Y} . \mathrm{T}$., and $\mathrm{Wu}, \mathrm{J}$. Y. (2011). Observation on therapeutic effect of Kangai injection combined with chemotherapy in 30 cases of advanced breast cancer. J. New Chin. Med. 43 (6), 88-89. doi:CNKI:SUN:REND.0.2011-06-046

Xiang, S. (2019). Clinical observation of Shenqi Fuzheng injection combined with chemotherapy in the treatment of breast cancer. Guangming J. Chin. Med. 34 (4), 619-621. doi:10.3969/j.issn.1003-8914.2019.04.051

Xiao, H. W. (2005). Shenqi-fuzheng injection combined with FEC in the treatment of breast carcinoma. J. Med. Theory Pract. 18 (8), 885-886. doi:10.3969/j.issn. 1001-7585.2005.08.007

Xie, F. (2014). Clinical analysis shenqifuzheng injection combined with chemotherapy in the treatment of breast cancer. Guide China Med. 12 (30), 19-20. doi:CNKI: SUN:YYXK.0.2014-30-013

Xie, R. D., and Long, F. (2019). Research progress of traditional Chinese medicine treatment of triple negative breast cancer. World Latest Med. Inform. 19 (28), 84-87. doi:10.19613/j.cnki.1671-3141.2019.28.036

Xiong, P., Lian, J., and Yu, X. Q. (2006). An electrocardiogram analysis in 83 galactophore cancer patients in ADM chemotherapy. Proc. Clin. Med. (4), 25-26. doi:10.3969/j.issn.1671-8631.2006.04.012

Xu, L., Zhu, H., and Bao, J. A. (2018). Analysis on factors associated with clinical medication of traditional Chinese medicine injections in a hospital in 2017. Anti-Infect. Pharm. 15 (10), 33-35. doi:10.13493/j.issn.1672-7878.2018. 10-007

Xu, Y. F., and Luo, X. L. (2019). Professor Luo Xiuli's clinical experience in treating triple-negative breast cancer based on data mining. Med. Inform. 32 (20), 159-160. doi:10.3969/j.issn.1006-1959.2019.20.050

Yang, B., Li, H. S., Qi, Y. C., and Lu, M. Y. (2007). Clinical study on treatment of mammary cancer by Shenqi fuzheng injection in cooperation with chemotherapy. Chin. J. Integr. Med. 13 (1), 37-40. doi:10.1007/s11655-007-0037-5

Yang, F. (2016). Study on the clinical application significance of Shenqi Fuzheng injection in adjuvant treatment of breast cancer. Nei Mongol J. Tradit. Chin. Med. 35 (5), 65. doi:10.3969/j.issn.1006-0979.2016.05.067

Yang, W. J. (2005). 30 cases of advanced breast cancer treated by Aidi injection combined with CAF chemotherapy. Jiangxi J. Tradit. Chin. Med. 36 (7), 46-47. doi:10.3969/j.issn.0411-9584.2005.07.030

Yang, X. L. (2008). Effect of Shenmai on Xinjiang Uygur breast cancer CAF chemotherapyinduced myocardial toxicity. Ürümqi, China: Xinjiang Medical University.

Yao, T. Z., Xu, Z. H., Ma, J. T., Zhang, N., Gao, W., Zhang, S. D., et al. (2016). Protection effect of Shenmai injection on myocardial damage induced by chemotherapy in postoperative breast cancer patients. Chin. J. Exp. Tradit. Med. Form. 22 (7), 208-212. doi:10.13422/j.cnki.syfjx.2016070208
Ye, X., Qiu, D. C., Dai, J. Y., Zhang, T., and Zhou, K. (2002). Clinical observation of Aidi injection combined with CAF regimen in the treatment of advanced breast cancer. J. Military Surgeon Southwest China. 4 (2), 11-12. doi:CNKI:SUN: JYXN.0.2002-02-006

Yin, X. D., Liu, X. D., Tong, J. D., Wang, Z., and Yuan, X. (1999). Analysis of the efficacy of Kanglaite injection combined with FACT in the treatment of 32 cases of breast cancer. Qilu J. Oncol. (2), 81-82. doi:CNKI:SUN:QLZL.0.199902-057

Yuan, J. W., Kong, C. B., Kang, G. Q., Liu, X. F., and Yang, S. J. (2008). Effects of neoadjuvant chemotherapy combined with shenqifuzheng injection on cell immune function for patients with breast cancer. Lishizhen Med. Mater. Medica Res. (5), 1099-1100. doi:10.3969/j.issn.1008-0805.2008.05.037

Zhai, X. J. (2014). Effect of compound kushen injection on toxic side effects and immune function of patients after breast cancer chemotherapy. Chin. J. Basic Med. Tradit. Chin. Med. 20 (6), 829-831. doi:CNKI:SUN:ZYJC.0.2014-06-046

Zhang, C. X. (2013). Observation of curative effect of Shenmai injection combined with CTF regimen on postoperative chemotherapy of breast cancer. Zhejiang J. Tradit. Chin. Med. 48 (4), 287-288. doi:10.3969/j.issn.0411-8421.2013.04.037

Zhang, H., and Zhang, H. (2019). Effect value of compound Kushen injection on curative effect and immune function of breast cancer patients undergoing chemotherapy. Guide China Med. 17 (27), 236-237. doi:CNKI:SUN:YYXK.0. 2019-27-197

Zhang, M. L., and Yang, H. (2009). AiDi injection combined with 5-fluorouracil plus oxaliplatin in the treatment of gastric cancer in the elderly. Pharm. Clin. Res. 17 (4), 328-330. doi:10.3969/j.issn.1673-7806.2009.04.016

Zhang, Z. D., Peng, W., and Chen, J. B. (2018). Effects of Shenqi fuzheng injection combined with CAF regimen on hematopoietic function, immune function and quality of life in patients with breast cancer. Chin. Archives Tradit. Chin. Med. 36 (8), 1904-1908. doi:10.13193/j.issn.1673-7717.2018.08.028

Zheng, Y., Wu, C. X., and Zhang, M. L. (2013). The epidemic and characteristics of female breast cancer in China. China Oncol. 2013 (8), 561-569. doi:10.3969/j. issn.1007-3969.2013.08.001

Zhou, S. Y., Zhang, S. J., Xu, H. Z., Sun, D. W., and Tang, J. H. (2017). Research progress of traditional Chinese medicine treatment of breast cancer. J. Basic Med. Tradit. Chin. Med. 23 (10), 1489-1492. doi:CNKI:SUN:ZYJC.0.201710-053

Zhu, C., and Sun, Y. P. (2012). Randomized controlled clinical study of Kangai injection combined with chemotherapy after breast cancer surgery. J. Pract. Tradit. Chin. Intern. Med. 26 (9), 61-63. doi:CNKI:SUN: SYZY.0.2012-09-037

Zhu, K., Li, K., and Dang, C. X. (2008). Effect of Shenqi Fuzheng injection on immune function of patients undergoing CEF chemotherapy after breast cancer surgery. J. Mod. Oncol. 16 (10), 1712-1714. doi:10.3969/j.issn.1672-4992.2008. 10.025

Zou, T. N., Nie, J. Y., and Chen, W. L. (2006). Attenuating effect of Shenqi Fuzheng Liquid on neoadjuvant chemotherapy for breast cancer. Chin. J. Pract. Ophthalmol. 21 (1), 75-77. doi:10.3969/j.issn.1001-1692.2006.01.026

Conflict of Interest: The authors declare that the research was conducted in the absence of any commercial or financial relationships that could be construed as a potential conflict of interest.

Copyright $\odot 2021$ Liu, Wang, Wang, Hu, Yang, Jin, Geng, Ni, Wu and Zhang. This is an open-access article distributed under the terms of the Creative Commons Attribution License (CC BY). The use, distribution or reproduction in other forums is permitted, provided the original author(s) and the copyright owner(s) are credited and that the original publication in this journal is cited, in accordance with accepted academic practice. No use, distribution or reproduction is permitted which does not comply with these terms. 


\section{GLOSSARY}

A Doxorubicin

ADRs Adverse reactions

ADI Aidi injection

BC Breast cancer

C Cyclophosphamide

E Epirubicin

F 5-Fluorouracil

chis Chinese herbal injections

CIs Confidence intervals

CR Complete response

CNKI The China National Knowledge Infrastructure Database

CKI Compound Kushen injection

HQI Huangqi injection

HCSI Huachansu injection

KAI Kangai injection
KLTI Kanglaite injection

KPS Karnofsky performance score

M Methotrexate

MDs Mean differences

NMA Network meta-analysis

ors Odds ratios

PLT Platelet

PR Partial response

RCTs Randomized controlled trials

SI Shengmai injection

SQFZI Shenqi Fuzheng injection

SMI Shenmai injection

SUCRA Surface under the cumulative ranking area curve

T Pirarubicin

TCM Traditional Chinese medicine

VIP The China Science and technology journal Database

WBC White blood cell 